\title{
Consumption of Sweet Beverages and Cancer Risk. A Systematic Review and Meta-Analysis of Observational Studies
}

\author{
Fjorida Llaha ${ }^{\dagger}$, Mercedes Gil-Lespinard ${ }^{\dagger}$, Pelin Unal ${ }^{(D}$, Izar de Villasante ${ }^{(D)}$, Jazmín Castañeda and \\ Raul Zamora-Ros *iD
}

check for updates

Citation: Llaha, F.; Gil-Lespinard, M.; Unal, P.; de Villasante, I.; Castañeda, J.; Zamora-Ros, R. Consumption of Sweet Beverages and Cancer Risk. A Systematic Review and Meta-Analysis of Observational Studies. Nutrients 2021, 13, 516. https://doi.org/ $10.3390 /$ nu13020516

Academic Editor: Emilio Sacanella Received: 30 December 2020

Accepted: 30 January 2021

Published: 4 February 2021

Publisher's Note: MDPI stays neutral with regard to jurisdictional claims in published maps and institutional affiliations.

Copyright: (c) 2021 by the authors. Licensee MDPI, Basel, Switzerland This article is an open access article distributed under the terms and conditions of the Creative Commons Attribution (CC BY) license (https:/ / creativecommons.org/licenses/by/ $4.0 /)$.
Unit of Nutrition and Cancer, Cancer Epidemiology Research Programme, Catalan Institute of Oncology (ICO), Bellvitge Biomedical Research Institute (IDIBELL), 08908 Barcelona, Spain; fllaha@idibell.cat (F.L.); mgill@idibell.cat (M.G.-L.); pelin.uenal@dkfz-heidelberg.de (P.U.); idevillasante@idibell.cat (I.d.V.); acastaneda@idibell.cat (J.C.)

* Correspondence: rzamora@idibell.cat; Tel.: +34-932607401

+ These authors contributed equally to this work.

\begin{abstract}
The consumption of sweet beverages, including sugar-sweetened beverages (SSB), artificialsweetened beverages (ASB) and fruit juices (FJ), is associated with the risk of different cardiometabolic diseases. It may also be linked to the development of certain types of tumors. We carried out a systematic review and meta-analysis of observational studies aimed at examining the association between sweet beverage intake and cancer risk. Suitable articles published up to June 2020 were sourced through PubMed, Web of Science and SCOPUS databases. Overall, 64 studies were identified, of which 27 were selected for the meta-analysis. This was performed by analyzing the multivariableadjusted $\mathrm{OR}, \mathrm{RR}$ or $\mathrm{HR}$ of the highest sweet beverage intake categories compared to the lowest one. Random effects showed significant positive association between SSB intake and breast (RR: 1.14, 95\% CI: 1.01-1.30) and prostate cancer risk (RR: 1.18, 95\% CI: 1.10-1.27) and also between FJs and prostate cancer risk (RR: 1.03, 95\% CI: 1.01-1.05). Although the statistically significant threshold was not reached, there tended to be positive associations for the following: SSBs and colorectal and pancreatic cancer risk; FJs and breast, colorectal and pancreatic cancer risk; and ASBs and pancreatic cancer risk. This study recommends limiting sweet beverage consumption. Furthermore, we propose to establish a homogeneous classification of beverages and investigate them separately, to better understand their role in carcinogenesis.
\end{abstract}

Keywords: systematic review; meta-analysis; cohort; case-control; sugar-sweetened beverages; artificial sweetened beverages; fruit juice; cancer

\section{Introduction}

The consumption of sweet beverages has increased in the last decades, with sugarsweetened beverages (SSB) and artificially sweetened beverages (ASB) among the most widely consumed [1,2]. SSBs contain high levels of sugar that usually come from added sucrose or high fructose corn syrup (HFCS). Another type of sweet beverage is fruit juice (FJ), including fresh and commercial FJs and nectars. Despite their natural and healthy image, they contain high levels of sugar in the form of fructose. Although whole fruit also contains fructose, the fiber present limits the insulin response and increases satiety [3]. High sugar consumption may contribute to excessive energy intake, leading to long-term weight gain [4], higher risk of type 2 diabetes [5] and cardiovascular disease [6].

It has been demonstrated that obesity and type 2 diabetes are well-known risk factors for cancer [7-9]. Diets high in added sugar usually result in weight gain and an increase in adiposity-related metabolic parameters, insulin resistance, bioactivity of steroid hormones, oxidative stress and inflammation, which finally leads to cancer development and progression [9]. The International Agency for Research on Cancer (IARC) reported as strong evidence that excess body fat is a major risk factor for many cancers, including esophageal, 
pancreatic, colorectal, post-menopausal breast, endometrial, renal, ovarian, gallbladder, hepatic and gastric cardia, among others [10].

High sugar intake impairs glucose and insulin tolerance and augments insulin and insulin-like growth factor (IGF) levels. Insulin and IGF are major determinants of proliferation and apoptosis, and may therefore influence carcinogenesis [11]. Beverages high in sugar, including SSBs and FJs, have high glycemic indexes [12] which is also suggested to be linked to cancer [13]. Moreover, both caloric and noncaloric sweet palatable substances have been demonstrated to activate the dopaminergic reward system. This can trigger addictive-like behaviors, which might be responsible for increased body fat [14]. ASBs contain low or non-caloric sweeteners (e.g., aspartame) and have been marked as healthier alternatives to SSBs. However, some studies have suggested that ASBs are also deleterious as regards obesity [15] and type 2 diabetes risk [5]. Moreover, it has also been suggested that long-term consumption of aspartame, used in many ASBs, might be carcinogenic [16]. Aspartame in liquids can quickly break down into methanol, and the subsequent metabolized formaldehyde is a documented carcinogenic substance [17].

In light of all this evidence, the association between consumption of sweet beverages and cancer risk has been investigated and reviewed by different studies. A meta-analysis from 2014 studied the association between SSB / ASB consumption and overall and specific cancer but no links were found [18]. Likewise, a 2019 meta-analysis did not find any significant association between SSB / ASB intake and pancreatic cancer risk [19]. However, the two mentioned studies did not perform a separate analysis of SSBs and ASBs which might have elucidated their particular role on cancer. A pooled analysis from 2012 [20] suggested a modest positive association between SSB intake and the risk of pancreatic cancer. Another similar study from 2010 [21] showed no significant association with colon cancer risk. A qualitative review of longitudinal studies from 2018 [22] reported inconsistent results for SSB/FJ intake and cancer risk. A recent French publication [23] reported a positive association between FJs and overall cancer risk. Regarding ASB intake, their results for breast, colorectal and prostate cancer risk were nonsignificant. However, another study [24] showed an increased risk for leukemia in the total population as well as for non-Hodgkin lymphoma and multiple myeloma in men only.

Evidence suggests that the link between sweet beverages consumption and cancer onset is biologically plausible. However, each type of beverage may have different mechanisms of action and different roles in cancer onset. Therefore, our study aimed to investigate these associations, by conducting separate analyses for SSB, ASB and FJ intake and cancer incidence. We analyzed case-control and cohort studies and performed a meta-analysis when feasible. Through this study we intend to update and develop a better understanding of the association between the consumption of sweet beverages and cancer incidence, a disease that caused 9.6 million deaths in 2018, a figure projected to nearly double by 2040 [25].

\section{Materials and Methods}

\subsection{Search Method for Identification of Studies}

This study was conducted according to the Preferred Reporting Items for Systematic Reviews Meta-Analysis (PRISMA) guidelines. To identify the suitable articles, we searched in PubMed, Web of Science and SCOPUS databases up to 31 June 2020, using the following keywords: ((((“soft drinks"[All Fields] OR "sugary drinks"[All Fields]) OR "sugary beverages"[All Fields]) OR "fruit juice"[All Fields]) OR "sugar-sweetened beverages"[MeSH Terms]) OR "artificially sweetened beverages"[MeSH Terms]) AND (((“"neoplasms"[MeSH Terms] OR "neoplasm"[All Fields]) OR "cancer"[All Fields]) OR "cancers"[All Fields]) OR "tumor"[All Fields]). We also applied search filters by article type (excluding books, reviews, systematic reviews and meta-analyses) and by species (including only humans). Moreover, reference lists of included manuscripts and relevant reviews were examined for any possible unidentified study. The search process was limited to English and Spanish languages. 


\subsection{Eligibility Criteria and Data Extraction}

Eligible cohort and case-control studies were selected if they met the following criteria: (1) included adult participants free of cancer (if prospective) or with no history of previous cancer (if case-control) at recruitment, except for nonmelanoma skin cancer; (2) overall or site-specific cancer incidence as an outcome; and (3) estimated and reported hazard ratio (HR), risk ratio (RR) or odds ratio (OR) with $95 \%$ confidence interval (CI) for the link between any type of sweet beverages and any type of cancer incidence. The exclusion criteria were: (1) participants with previous cancer history or currently undergoing cancer treatment; (2) cancer survival and cancer mortality as an outcome; and (3) duplicated studies. The following data were extracted: first author's name, publication year, study name, country, age and sex of the participants, study sample size, number of cases and controls, follow-up duration, cancer site, type of exposure and amount of intake, dietary assessment methods, confounders' adjustment and HR/RR/OR with $95 \% \mathrm{CI}$ for the larger degree of adjustment. When time-varying results were reported, those related to baseline data were extracted.

Three review authors independently performed the literature search, study selection and data extraction (FL, MG-L, and PU). Disagreements were discussed between all authors until a consensus was reached.

\subsection{Quality Assessment of Included Studies}

Two independent review authors (FL and MG-L) examined the methodological quality of the individual studies using the Risk Of Bias In Non-randomized Studies-of Exposures (ROBINS-E) [26] tool for cohort studies and the Newcastle-Ottawa Scale (NOS) [27] adapted for case-control studies. The ROBINS-E tool evaluates the risk of bias by assessing different domains: confounding variables, selection of participants into the study, classification of exposures, departures from intended exposures, missing data, measurement of outcomes and selection of the reported result. Low, moderate or serious risk of bias was established in each study considering all domains. The NOS assesses the selection of groups (0-4 stars), adequacy of comparability between groups (adjustment for confounders) ( $0-2$ stars) and ascertainment of the exposure of interest for case-control studies (0-3 stars). For selection domain, we considered studies with $0-1,2-3$ and 4 stars as serious bias risk, moderate bias risk and high-quality risk, respectively. For comparability between groups, we considered those with 0,1 and 2 as serious, moderate, and low bias risk, respectively. And finally, for ascertainment of exposure, we considered those $0,1-2$ and 3 as serious risk, moderate risk and low bias risk, respectively. In both tools, when data were not enough for judgment, the domain was classified as 'no information'.

\subsection{Data Synthesis and Statistical Analysis}

The first obstacle that we had to overcome was the lack of a unique definition for beverages and a variety of other terms. In this text, the following group terms are used to generalize these products: SSB for sugar-sweetened beverages (regular soft drinks/sodas, and non-diet soft drinks/sodas), ASB for artificially sweetened beverages (low and noncaloric soft drinks/sodas, and diet soft drinks/sodas) and FJ for fruit juices. In addition, two other terms are used: SB for sweetened beverages that includes both SSBs and ASBs; SFJ for high-sugar (added or natural) beverages that includes both SSBs and FJs. The quantity of each beverage was provided mostly as categories of frequency of consumption, either in amount ( $\mathrm{mL}$ or $\mathrm{g} /$ day) or serving sizes (cans for SSBs and ASBs, glasses for FJs). To unify the data, we converted the categories to $\mathrm{mL} /$ day, based on the study-specific serving size for each beverage. When the serving size was not reported, we referred the national data of each study. Thus, we considered one can equal to $330 \mathrm{~mL}$ and one glass equal to $200 \mathrm{~mL}$ for European countries [28], one can equal to $360 \mathrm{~mL}$ and one glass equal to $240 \mathrm{~mL}$ for the United States [29], and one can equal to $375 \mathrm{~mL}$ for Australia [30]. One US study [31] expressed consumption as grams of sugar, and we weighed up an average of $10.5 \mathrm{~g}$ of sugar per $100 \mathrm{~mL}$ of SSB and an average of $9.6 \mathrm{~g}$ of sugar per $100 \mathrm{~mL}$ of FJ. This 
was calculated based on the sugar content of different commercially available products of popular brands [32].

Prior to the analysis, the selected studies were classified by outcome (cancer incidence by site) and exposure (SB, SSB, ASB, FJ and SFJ). Data were summarized in a narrative manner and a meta-analysis was performed only if at least three studies reported data for the same exposure and outcome. In the meta-analysis, results for the total number of participants were considered. Separate analyses were considered (e.g., European-American and African-American women) when the article did not report indices for total population. In the same manner, if studies reported data for specific beverages (e.g., caffeinated and noncaffeinated SSBs), results for the total beverage group (e.g., total SSBs) were weighted up. Despite having extracted data on fruit and vegetables juices together, for the meta-analysis we considered the studies that indicated FJs as the predominant beverage consumed. The meta-analysis was performed by pooling the multivariable-adjusted RR/HR/OR of the highest category of the exposure versus the lowest one, and random effects models were assumed. If statistical outliers were identified, secondary analyses were performed (without outliers) to remove possible sources of heterogeneity. An outlier was considered when its $95 \%$ CI lied outside the $95 \%$ CI of the pooled effect. To further explain heterogeneity, we performed subgroup and sensitive analyses, dividing studies according to design (cohort/case-control), country (US/non-US, mostly European), level of overall risk of bias (serious/low-moderate) and beverage intake category (high vs. non-consumer/high vs. low). We used Cochran's $Q, I^{2}$ and Tau ${ }^{2}$ statistics to measure between-study heterogeneity. The statistical analysis was performed with the Metafor package [33] of the R software, version 4.0.1. $P$ values $<0.05$ were considered statistically significant.

\section{Results}

\subsection{Literature Search and Study Characteristics}

The study selection process according to PRISMA guidelines is reported in Figure 1. In total, 869 potential publications were identified from the databases (PubMed, Web of Science and SCOPUS) and other sources. After removing duplicates, 596 articles were selected, from which 435 were excluded based on titles and 26 on abstracts. Of 135 eligible articles, 71 were excluded due to the following reasons: 59 did not report risk index for sweet beverages and cancer incidence, 3 full-texts were not available, 7 considered other outcomes, 1 case-control study included controls with cancer at recruitment and 1 publication was not in English or Spanish. Finally, 64 studies were included in the systematic review, 27 cohort [23,24,28,31,34-56] and 37 case-control studies [57-93]. Of these, 27 studies were meta-analyzed.

Of the included studies, 29 were performed in the United States (US), 17 in Europe, 6 in Asia, 5 in Canada, 3 in Australia, 2 in Latin-America, 1 in Egypt and 1 was multinational (Italy, Spain, Poland, Northern Ireland, India, Cuba, Canada, Australia and Sudan). They usually included both male and female participants. Ages ranged from 18 to 97 years. The 27 cohort studies were published between 2003 and 2020 and enrolled 4,458,056 participants in total, of which 30,646 developed cancer. Mean duration of the follow-up in cohort studies varied from 2 to 20 years. The 37 case-control studies were published between 1985 and 2019. In total, they enrolled 20,827 cancer cases and 34,315 controls. Most of the controls were selected from the general population. 


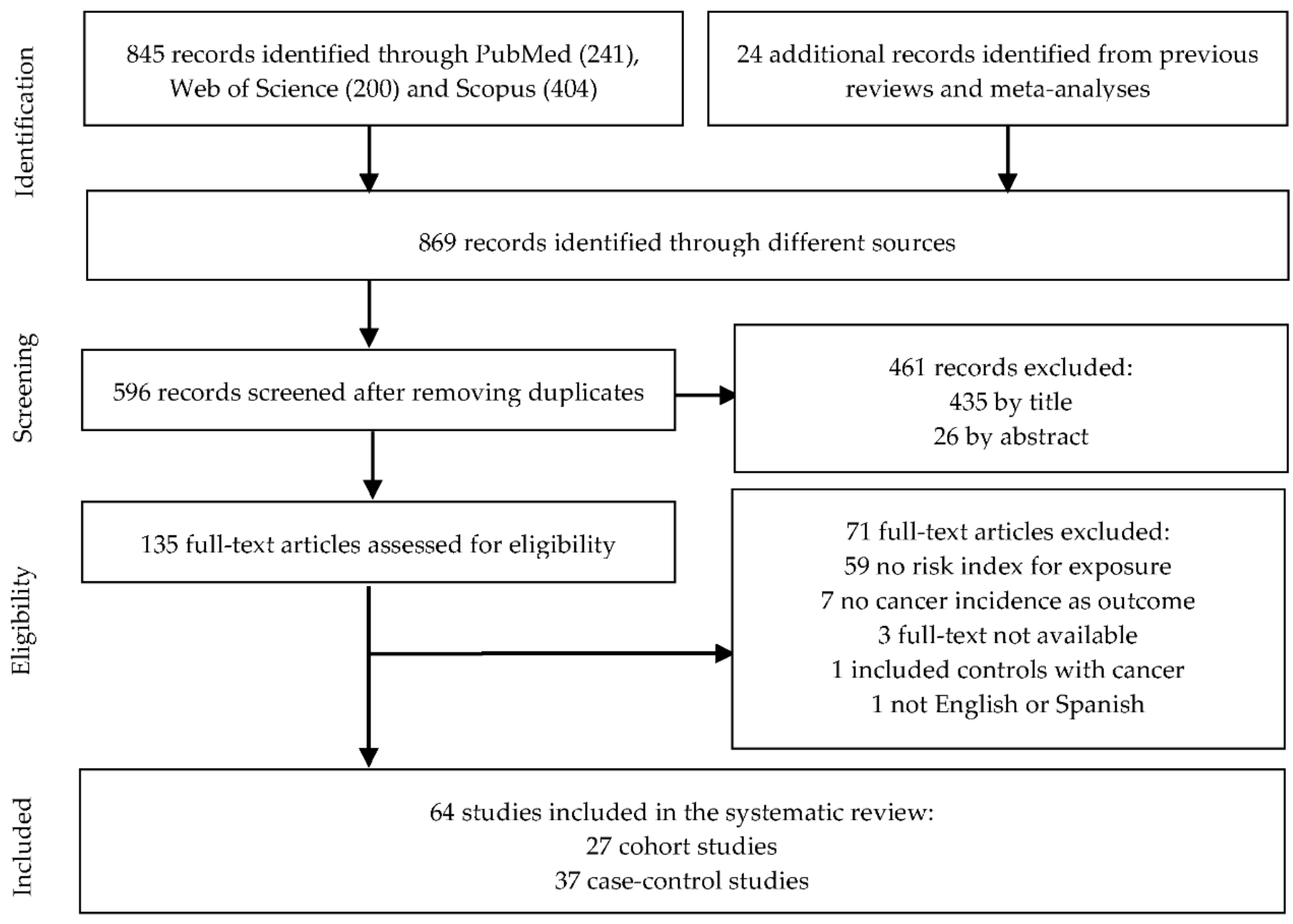

Figure 1. Prisma diagram.

Sweet beverage consumption in both cohort and case-control studies was expressed as categorical or continuous variables. Exposure assessment was collected using food frequency questionnaires (FFQ), 24-h dietary recalls (24-H DR), dietary questionnaires (DQ), interviews, or surveys. Among all the studies, 37 types of cancer were considered as an outcome and 4 cohorts reported data for overall cancer risk, including different types of cancer $[23,50,52,54]$. In most of the studies, the outcome was confirmed by a medical diagnosis. Overall characteristics of the included studies are summarized in Table 1. Results of the meta-analysis for the random-effect model are summarized in Table 2 and for the subgroup analysis in Table S1. 
Table 1. Overall characteristic of the included studies.

\begin{tabular}{|c|c|c|c|c|c|c|c|c|c|c|c|}
\hline \multirow[b]{2}{*}{ Source } & \multirow[b]{2}{*}{ Country, Study Name } & \multirow[b]{2}{*}{ Cancer Type } & \multirow[b]{2}{*}{ Study Design } & \multirow[b]{2}{*}{$\begin{array}{l}\text { Population Follow-Up } \\
\text { (Years) }\end{array}$} & \multicolumn{4}{|c|}{ Breast Cancer (Breast, Pre- and Post-Menopausal) } & \multirow[b]{2}{*}{$\underset{+}{\text { Type and Amount of Beverages Intake }}$} & \multirow[b]{2}{*}{ 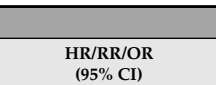 } & \multirow[b]{2}{*}{ Adjustments } \\
\hline & & & & & Cases & $\begin{array}{l}\text { Age (Mean/SD } \\
\text { or Range) }\end{array}$ & $\operatorname{sex}(\%)$ & $\begin{array}{l}\text { Dietary Assessment } \\
\text { Method }\end{array}$ & & & \\
\hline $\begin{array}{l}\text { Chandran et al., } 2006 \\
[57]]\end{array}$ & $\begin{array}{l}\text { US, } \\
\text { WCHS }\end{array}$ & $\begin{array}{l}\text { Breast } \\
\text { Pre-M } \\
\text { Post-M }\end{array}$ & PB case-control & 3148 & $\begin{array}{l}797 \\
761\end{array}$ & $20-75$ & $F(100)$ & 125-item FFQ & $\begin{array}{l}\text { SSB: } \geq 152 \text { vs. }<152 \mathrm{~mL} / \text { day } \\
\text { SSB: } \geq 152 \text { vs. }<152 \mathrm{~mL} / \text { day } \\
\text { SSB: } \geq 152 \text { vs. }<152 \mathrm{~mL} / \text { day }\end{array}$ & $\begin{array}{l}\text { OR: } 0.97(0.74-1.27) \text { (AA) } \\
\text { OR: } 1.31(0.91-1.89) \text { (EA) } \\
\text { OR } 1.17(0.79-1.74) \text { (AA) } \\
\text { OR: } 0.95(0.58-1.56 \text { ) (EA) } \\
\text { OR: } 0.76(0.51-1.12) \text { (AA) } \\
\text { OR: } 2.05(1.13-3.7) \text { (EA) }\end{array}$ & $\begin{array}{l}\text { Age, ethnicity, country, } \\
\text { education, age at menarche, } \\
\text { menopause and first birth, } \\
\text { MS, parity, BF status, history } \\
\text { of benign breast disease, } \\
\text { family history of BC, HRT, OC } \\
\text { use, BMI and study site. }\end{array}$ \\
\hline $\begin{array}{c}\text { Chazelas et al., } 2019 \\
\text { [23] }\end{array}$ & $\begin{array}{l}\text { France, } \\
\text { NNS }\end{array}$ & Post-M & Cohort & $\begin{array}{l}101,257 \\
5.1 \text { (median) }\end{array}$ & 283 & 42.2/14.4 & $\mathrm{F}(78)$ & 24H-DR & 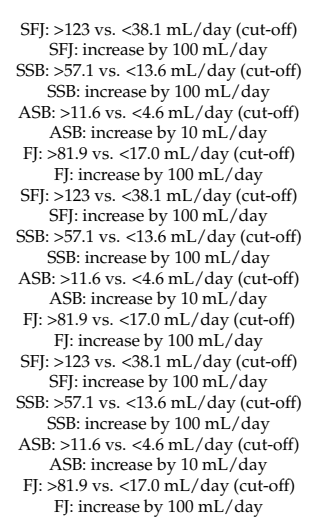 & 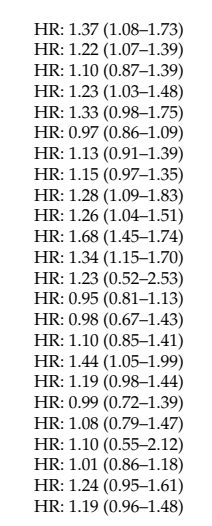 & $\begin{array}{l}\text { Smoking, education, PA, BMI, } \\
\text { and height. }\end{array}$ \\
\hline $\begin{array}{c}\text { Hirvonen et al., } 2006 \\
{[51]}\end{array}$ & France, SUVIMAX & Breast & Cohort & $\begin{array}{l}4396 \\
6.6\end{array}$ & 95 & $35-60$ & $F(100)$ & 24H-DR & F: $>150 \mathrm{~mL} /$ day vs. none & RR: $1.29(0.80-2.09)$ & $\begin{array}{l}\text { Age, smoking, number of } \\
\text { children, OC use, family } \\
\text { history of } \mathrm{BC} \text {, and MS. }\end{array}$ \\
\hline $\begin{array}{c}\text { Makarem et al., } 2018 \\
{[52]}\end{array}$ & us & Breast & Cohort & $\begin{array}{c}3184 \\
4\end{array}$ & 128 & 54.3 & F (53) & FFQ & 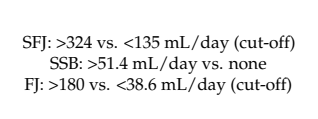 & $\begin{array}{l}\text { HR: } 1.00(0.65-1.57) \\
\text { HR: } 1.04(0.64-1.71) \\
\text { HR: } 1.03(0.67-1.62)\end{array}$ & 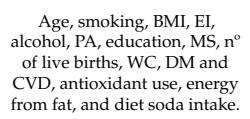 \\
\hline $\begin{array}{c}\text { Marzbani et al., } 2019 \\
{[58]}\end{array}$ & Iran & Breast & HB case-control & 620 & 212 & 40.2 & $F(100)$ & $\begin{array}{c}\text { 11-item healthcare } \\
\text { form }\end{array}$ & $\begin{array}{c}\mathrm{SB}^{7}: \text { favorable intake vs. } \leq 1 \\
\text { time /month }\end{array}$ & OR: $2.8(1.9-4.3)$ & Age, education, and BMI \\
\hline $\begin{array}{l}\text { McLaughlin et al., } 1992 \\
{[69]}\end{array}$ & us & Breast & PB case-control & 3234 & 1617 & 56.7 & $\mathrm{~F}(100)$ & SQ-interview & $\mathrm{SB}^{2}$ : ever vs. never & OR: $1.08(0.92-1.26)$ & $\begin{array}{l}\text { Age, alcohol, country, race, } \\
\text { MS, age at first live birth, } \\
\text { diagnosis of benign cancers, } \\
\text { and family history of BC. }\end{array}$ \\
\hline $\begin{array}{c}\text { Potischman et al., } 2002 \\
{[80]}\end{array}$ & us & Breast & PB case-control & 2019 & 568 & $20-44$ & $\mathrm{~F}(100)$ & 100-item FFQ & $\mathrm{SSB}: \geq 320 \mathrm{~mL} /$ day vs. none & OR: $1.09(0.8-1.5)$ & $\begin{array}{l}\text { Age at diagnosis, study site, } \\
\text { race, education, alcohol } \\
\text { consumption, years of OC } \\
\text { use, smoking, BMI, and EI. }\end{array}$ \\
\hline
\end{tabular}


Table 1. Cont.

\begin{tabular}{|c|c|c|c|c|c|c|c|c|c|c|c|}
\hline $\begin{array}{l}\text { Romanos-Nanclares } \\
\text { et al, } 2019[53]\end{array}$ & Spain & $\begin{array}{l}\text { Pre-M } \\
\text { Post-M }\end{array}$ & Cohort & $\frac{10,713}{2}$ & 43 & 33.0 (median) & $\mathrm{F}(100)$ & FFQ & $\begin{array}{l}\text { SSB: }>47.1 \text { vs. }<11 \mathrm{~mL} / \text { day } \\
\text { SSB: } \geq 11 \mathrm{~mL} / \text { day vs. none } \\
\text { SSB: }>47.1 \text { vs. }<11 \mathrm{~mL} / \text { day }\end{array}$ & $\begin{array}{l}\text { HR: } 1.36(0.74-2.50) \\
\text { HR: } 1.16(0.66-2.07) \\
\text { HR: } 2.12(1.01-4.41)\end{array}$ & $\begin{array}{l}\text { Age, height, family history of } \\
\text { BC, hsmoking, PA, BMI, age at } \\
\text { menarche and menopause, } \\
\text { MS, HRT, number of } \\
\text { pregnancies } 6 \text { month and } \\
\text { before } 30 \text { years old, months of } \\
\text { BF, accohol, education, DM, } \\
\text { GI, EI, U-P food and coffee } \\
\text { consumption, and Med-diet } \\
\text { adherence. }\end{array}$ \\
\hline Hodge et al., 2018 [54] & Australia, MCCS & Post-M & Cohort & $\begin{array}{c}35,593 \\
19\end{array}$ & 946 & 54.6 & $\mathrm{~F}(100)$ & 121-item FFQ & $\begin{array}{l}\text { SSB: }: 200 \mathrm{vs},<6.7 \mathrm{~mL} / \text { day } \\
\text { ASB: } \geq 200 \mathrm{vs} .<6.7 \mathrm{~mL} / \mathrm{day}\end{array}$ & $\begin{array}{l}\text { HR: } 1.11(0.85-1.45) \\
\text { HR: } 0.95(0.73-1.25)\end{array}$ & $\begin{array}{l}\text { Socioeconomic indexes, } \\
\text { country of birth, alcohol } \\
\text { intake, smoking, PA, } \\
\text { Med-diet score, and sex. ASB } \\
\text { also for SSB consumption and } \\
\text { WC. } \\
\end{array}$ \\
\hline Nomura et al., 2016 [55] & $\begin{array}{l}\text { US, } \\
\text { BWHS }\end{array}$ & $\begin{array}{l}\text { Breast } \\
\text { Pre-M } \\
\text { Post-M }\end{array}$ & Cohort & $\begin{array}{c}49,103 \\
13.8\end{array}$ & $\begin{array}{l}1827 \\
678 \\
826\end{array}$ & $21-69$ & $\mathrm{~F}(100)$ & FFQ & 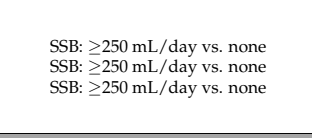 & $\begin{array}{l}\text { HR: }: .71(0.50-1.02) \\
\text { HR: } 1.72(0.191-23) \\
\text { HR: } 1.11((0.77-1.61)\end{array}$ & $\begin{array}{l}\text { Age, geographic region of } \\
\text { residence, EII, smoking, family } \\
\text { history of BC, education, MS, } \\
\text { OC use, parity, HRT, BMI, } \\
\text { alcohol, PA, and sedentary } \\
\text { time. }\end{array}$ \\
\hline & \multicolumn{11}{|c|}{ Colorectal and Rectal Cancer } \\
\hline Source & Country, Study Name & Cancer Type & Study Design & $\begin{array}{c}\begin{array}{c}\text { Population Follow-Up } \\
\text { (Years) }\end{array} \\
\end{array}$ & Cases & $\begin{array}{c}\text { Age (Mean/SD } \\
\text { or Range) }\end{array}$ & Sex $(\%)$ & $\begin{array}{c}\text { Dietary Assessment } \\
\text { Method }\end{array}$ & $\begin{array}{c}\text { Type and Amount of Beverages } \\
\text { Intake } \text { + }^{+}\end{array}$ & $\begin{array}{c}\mathrm{HR} / \mathrm{RR} / \mathrm{OR} \\
(95 \% \mathrm{CI})\end{array}$ & Adjustments \\
\hline $\begin{array}{l}\text { Bener et al., } \\
2010[88]\end{array}$ & Qatar & Colorectal & HB case-control & 428 & 146 & 53.4 & $\mathrm{M}(58)$ & DQ & SB: $\geq 330$ vs. $\leq 47.1 \mathrm{~mL} /$ day & OR: $1.62(1.19-2.17)$ & Not reported \\
\hline $\begin{array}{l}\text { Chazelas et al., } 2019 \\
\text { [23] }\end{array}$ & France & Colorectal & Cohort & $\begin{array}{l}101,257 \\
5.1 \text { (median) }\end{array}$ & 166 & $42.2(14.4)$ & $\mathrm{F}(78)$ & $24 \mathrm{H}-\mathrm{DR}$ & 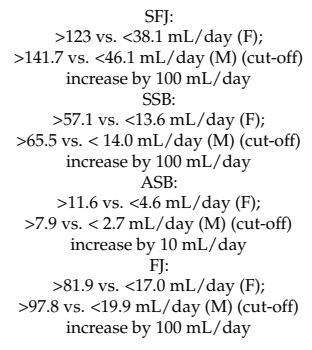 & $\begin{array}{l}\text { HR: } 1.07(0.63-1.80) \\
\text { HR: } 1.10(0.84-1.46) \\
\text { HR: } 1.01(0.59-1.71) \\
\text { HR: } 1.11(0.72-1.71) \\
\text { HR: } 0.80(0.44-1.46) \\
\text { HR: } 1.02(0.94-1.10) \\
\text { HR: } 1.19(0.78-1.82) \\
\text { HR: } 1.05(0.75-1.46)\end{array}$ & $\begin{array}{l}\text { Smoking, education, PA, BMI, } \\
\text { and height. }\end{array}$ \\
\hline Hodge et al, 2018 [54] & Australia, MCCS & Colorectal & Cohort & $\begin{array}{c}35,593 \\
19\end{array}$ & 1055 & 54.6 & $\mathrm{M} / \mathrm{F}$ & 121-item FFQ & $\begin{array}{l}\text { SSB: } \geq 200 \mathrm{vss}<6.7 \mathrm{~mL} / \mathrm{day} \\
\text { ASB: } \geq 200 \mathrm{vs}<<6.7 \mathrm{~mL} / \mathrm{day}\end{array}$ & $\begin{array}{l}\text { HR: } 1.28(1.04-1.57) \\
\text { HR: } 0.79(0.60-1.06)\end{array}$ & $\begin{array}{l}\text { Socioeconomic indexes, } \\
\text { country, alcohol, smoking, } \\
\text { MA, } \\
\text { ald-diet score, and sex. ASB } \\
\text { also for SSB consumption and } \\
\text { WC. } \\
\end{array}$ \\
\hline $\begin{array}{c}\text { Makarem et al., } 2018 \\
{[52]}\end{array}$ & us & Colorectal & Cohort & $\begin{array}{l}3184 \\
4\end{array}$ & 68 & 54.3 & F (53) & FFQ & $\begin{array}{l}\text { SFF: }>362.6 \mathrm{vs} .<154.3 \mathrm{~mL} / \text { day (cut-off) } \\
\text { SSB: }>180 \mathrm{vs}<25.7 \mathrm{~mL} / \text { /ay (cut-off) } \\
\text { FJ: }>180 \mathrm{vs.}<<88.9 \mathrm{~mL} / \text { day (cut-off) }\end{array}$ & $\begin{array}{l}\text { HR: } 1.39(0.68-2.82) \\
\text { HR } 0.96(0.51-.82) \\
\text { HR: } 1.66(0.88-3.12)\end{array}$ & 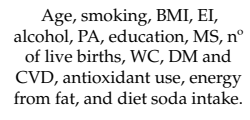 \\
\hline $\begin{array}{c}\text { Mahfouz et al., } 2014 \\
{[89]}\end{array}$ & Egypt & Colorectal & HB case-control & $\begin{array}{c}450 \\
1 \\
\end{array}$ & 150 & $<20->60$ & $\mathrm{~F}(52)$ & $\mathrm{DQ}$ & $\begin{array}{l}\text { SB: daily vs. not daily } \\
\text { FJ: daily vs. not daily }\end{array}$ & $\begin{array}{l}\text { OR: } 4.6(1.9-11.01) \\
\text { OR: } 0.18(0.09-0.36) \\
\end{array}$ & Not reported \\
\hline
\end{tabular}


Table 1. Cont.

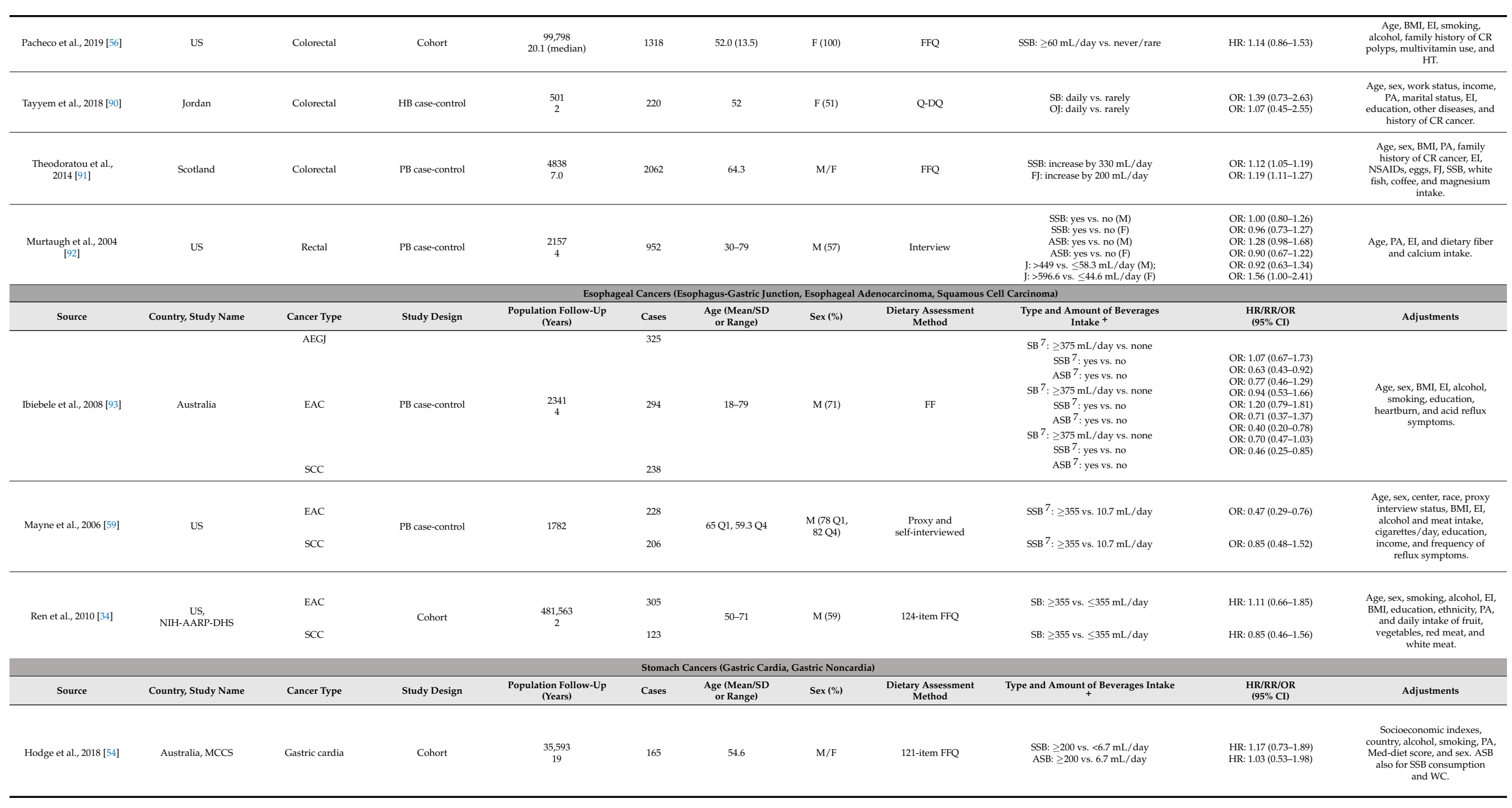


Table 1. Cont.

\begin{tabular}{|c|c|c|c|c|c|c|c|c|c|c|c|}
\hline Mayne et al., 2006 [59] & us & $\begin{array}{c}\text { Gastric cardia } \\
\text { Gastric noncardia }\end{array}$ & PB case-control & 1782 & $\begin{array}{l}255 \\
352 \\
\end{array}$ & $65 \mathrm{Q} 1,59.3 \mathrm{Q} 4$ & $\begin{array}{l}\mathrm{M}(78 \mathrm{Q} 1 \\
82 \mathrm{Q} 4)\end{array}$ & $\begin{array}{c}\text { Proxy and } \\
\text { self-interviewed }\end{array}$ & $\begin{array}{l}\operatorname{SSB}^{7}: \geq 355 \text { vs. }<10.7 \mathrm{~mL} / \text { day } \\
\operatorname{SSB}^{7}: \geq 355 \text { vs. }<10.7 \mathrm{~mL} / \text { day }\end{array}$ & $\begin{array}{l}\text { OR: } 0.74(0.46-1.16) \\
\text { OR: } 0.65(0.43-0.98) \\
\end{array}$ & $\begin{array}{l}\text { Age, sex, center, race, proxy } \\
\text { interview status, BMI, EI, } \\
\text { alcohol and meatitintake } \\
\text { cigaretes/ day, eduction, } \\
\text { incomes, and frequency of } \\
\text { reflux symptoms. }\end{array}$ \\
\hline Ren et al., 2010 [34] & $\begin{array}{l}\text { US, } \\
\text { NIH-AARP-DHS }\end{array}$ & $\begin{array}{c}\text { Gastric cardia } \\
\text { Gastric noncardia }\end{array}$ & Cohort & $\underset{2}{481,563}$ & 231 & $50-71$ & M (59) & 124-item FFQ & $\mathrm{SB}: \leq 355$ vs. $\geq 355 \mathrm{~mL} /$ day & HR: $0.89(0.55-1.45)$ & $\begin{array}{l}\text { Age, sex, smoking, alcohol, } \mathrm{EI}, \\
\text { BMI, education, ethnicity, } \mathrm{PA} \\
\text { and daily intake of fruit, } \\
\text { vegetables, and white meat. }\end{array}$ \\
\hline \multicolumn{12}{|c|}{ Pancreatic Cancer } \\
\hline Source & Country, Study Name & Cancer Type & Study Design & 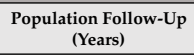 & Cases & $\begin{array}{l}\text { Age (Mean/SD } \\
\text { or Range) }\end{array}$ & Sex $(\%)$ & $\begin{array}{c}\text { Dietary Assessment } \\
\text { Method }\end{array}$ & Type and Amount of Beverages Intake & $\begin{array}{l}\mathrm{HR} / \mathrm{RR} / \mathrm{OR} \\
(95 \% \mathrm{CI})\end{array}$ & Adjustments \\
\hline Bao et al., $2008[42]$ & $\begin{array}{c}\text { US, } \\
\text { NIH-AARP-DHS }\end{array}$ & Pancreatic & Cohort & $\begin{array}{c}487,922 \\
7.2\end{array}$ & 1258 & $50-71$ & $\mathrm{~F}(41)$ & 124-item FFQ & $\begin{array}{l}\text { SB: } 816.9 \mathrm{~mL} / \text { day (median) vs. none } \\
\text { SSB: } 152.8 \mathrm{~mL} \text { dday (median) v. none } \\
\text { ASB: } 816.9 \mathrm{~mL} / \text { day (median) vs. none }\end{array}$ & $\begin{array}{l}\text { RR: } 1.07(0.86-1.33) \\
\text { RR: } 1.01(0.77-1.31) \\
\text { RR: } 1.11(0.86-1.44)\end{array}$ & $\begin{array}{l}\text { Age, sex, race, education, } \\
\text { BMI, alcohol, smoking, PA, EI, } \\
\text { and foliate intake. SSB and } \\
\text { ASB were mutually adjusted. }\end{array}$ \\
\hline Chan et al., 2009 [76] & $\begin{array}{l}\text { US, } \\
\text { SFB }\end{array}$ & Pancreatic & PB case-control & 2233 & 532 & $21-85$ & M (53) & 131-item FFQ & $\begin{array}{l}\mathrm{SB}: \geq 355 \mathrm{~mL} / \text { day vs. none } \\
\mathrm{SB}^{7}: \geq 355 \mathrm{~mL} / \text { day vs. none } \\
\mathrm{SSB}^{7}: \geq 355 \mathrm{~mL} / \text { day vs. none } \\
\mathrm{ASB} 7: \geq 355 \mathrm{~mL} / \text { day vs. none } \\
\mathrm{SSB}^{2}: \geq 355 \mathrm{~mL} / \text { day vs. none }\end{array}$ & $\begin{array}{l}\text { OR: } 1.0(0.7-1.3) \\
\text { OR: } 1.1(0.8-1.5) \\
\text { OR: } 0.9(0.6-1.3) \\
\text { OR: } 1.5(1.1-2.1) \\
\text { OR: } 1.0(0.6-1.8)\end{array}$ & $\begin{array}{l}\text { Age, sex, EI, BMI, race, } \\
\text { education, smoking, history } \\
\text { of DM, PA, red and white } \\
\text { meat, fruit and vegetables, } \\
\text { eggs, dairy, whole and refine } \\
\text { grained, and sweets. SSB and } \\
\text { ASB were mutually adjusted. }\end{array}$ \\
\hline Gallus et al, 2011 [77] & Italy & Pancreatic & HB case-control & $\begin{array}{c}978 \\
7\end{array}$ & 326 & 63 (median) & M (53) & $\mathrm{FFQ}$ & $\mathrm{SB}^{7}: \geq 150 \mathrm{vs} .<150 \mathrm{~mL} / \mathrm{day}$ & OR: $1.02(0.72-1.44)$ & $\begin{array}{l}\text { Age, sex, study center, } \\
\text { education, BMI, smoking, } \\
\text { alcohol, EI, family history of } \\
\text { pancreatic cancer, and DM. }\end{array}$ \\
\hline Gold et al., 1985 [78] & us & Pancreatic & $\mathrm{HB}, \mathrm{PB}$ case-control & 676 & 274 & 66.1 & $\mathrm{~F}(53)$ & Interview & ASB: ever vs. never & OR: $0.66(0.38-1.2)$ & $\begin{array}{l}\text { Religion, occupation, } \\
\text { smoking, and alcohol. }\end{array}$ \\
\hline $\begin{array}{l}\text { Lyon et al., } \\
1992[79]\end{array}$ & us & Pancreatic & PB case-control & 512 & 149 & $40-79$ & $\mathrm{M} / \mathrm{F}$ & DQ & SB (caff): ever vs. never & OR: $1.31(0.89-1.94)$ & Unadjusted. \\
\hline Mack et al., 1986 [81] & us & Pancreatic & PB case-control & 980 & 490 & $18-65$ & $\mathrm{M}(58)$ & $\begin{array}{l}\text { Proxy and direct } \\
\text { Interview }\end{array}$ & $\mathrm{SB}^{7}: \geq 1650$ vs. $<1320 \mathrm{~mL} /$ day & RR: $2.6(0.9-7.4)$ & Not reported \\
\hline Mueller et al., $2010[43]$ & $\begin{array}{l}\text { China and Singapore, } \\
\text { SCHS }\end{array}$ & Pancreatic & Cohort & $\begin{array}{c}60,524 \\
14\end{array}$ & 140 & 56.5 & $F(56)$ & $\mathrm{FFQ}$ & $\begin{array}{l}\text { SB: } \geq 67.7 \mathrm{~mL} / \text { day vs. none } \\
\mathrm{J}^{5}: \geq 67.7 \mathrm{~mL} / \text { day vs. none }\end{array}$ & $\begin{array}{l}\text { HR: } 1.87(1.10-3.15) \\
\text { HR: } 1.31(0.74-2.30)\end{array}$ & $\begin{array}{l}\text { Age, sex, smoking, BMI, } \\
\text { acohol, EI, PA, DM, } \\
\text { education, added sugar, and } \\
\text { candy. SB and J were } \\
\text { mutually adjusted. }\end{array}$ \\
\hline $\begin{array}{l}\text { Nothlings et al., } 2007 \\
{[44]}\end{array}$ & us & Pancreatic & Cohort & $\frac{162,150}{8}$ & 434 & 59.8 & $\mathrm{~F}(55)$ & $\mathrm{FFQ}$ & $\begin{array}{c}\text { SSB: } \geq 151.4 \mathrm{~mL} / 2000 \mathrm{kcal} / \mathrm{day} \text { vs. } \\
\text { Fone } \\
\text { FJ: } \geq 120 \mathrm{vs} .<9.4 \mathrm{~mL} / 2000 \mathrm{kcal} / \mathrm{day}\end{array}$ & $\begin{array}{l}\text { RR: } 1.07(0.82,1.41) \\
\text { RR: } 1.08(0.83,1.41)\end{array}$ & $\begin{array}{l}\text { Age, sex, smoking, BMI, EI, } \\
\text { time on study, race, family } \\
\text { history of pancreatic cancer, } \\
\text { intake of red, and processed } \\
\text { meat. }\end{array}$ \\
\hline $\begin{array}{l}\text { Navarrete-Muñoz et al., } \\
2016[45]\end{array}$ & $\begin{array}{c}10 \text { European countries } \\
\text { †, EPIC }\end{array}$ & Pancreatic & Cohort & $\begin{array}{c}477,206 \\
11.4\end{array}$ & 865 & 51 & $F(70)$ & DQ-country specific & $\begin{array}{l}\text { SB: > } 196.4 \text { vs. } 0.1-13.1 \mathrm{~mL} / \text { day } \\
\text { SB: increase by } 100 \mathrm{~mL} / \text { day } \\
\text { SSB: }>121.4 \text { vs. } 0.1-4.5 \mathrm{~mL} / \text { day } \\
\text { SSBB: increase by } 100 \mathrm{~mL} / \text { day } \\
\text { ASB : }>92.2 \text { vs } 0.1-2.0 \mathrm{~mL} / \text { day } \\
\text { ASB: increase by } 10 \mathrm{~mL} / \text { day } \\
\mathrm{FJ}^{6}:>123.1 \text { vs. } 0.1-8.3 \mathrm{~mL} / \text { day } \\
\mathrm{FJ}^{6} \text { : increase by } 100 \mathrm{~mL} / \text { day }\end{array}$ & $\begin{array}{l}\text { HR: } 0.90(0.68-1.19) \\
\text { HR: } 1.02(0.98-1.06) \\
\text { HR: }: .90(0.65-1.25) \\
\text { HR } 1.02(0.97-1.08) \\
\text { HR: } 0.99(0.61-1.0) \\
\text { HR: } 1.02(0.96-1.08) \\
\text { HR } 0.74 \text { (0.5.7-.97) } \\
\text { HR: } 0.91(0.84-0.98)\end{array}$ & $\begin{array}{l}\text { Age, sex, smoking, BMI, } \\
\text { alcohol, EI, study center, PA, } \\
\text { and DM. FJ and SB were } \\
\text { mutually adjusted. }\end{array}$ \\
\hline $\begin{array}{l}\text { Schernhammer et al., } \\
2005[46]\end{array}$ & $\begin{array}{l}\text { US, } \\
\text { HPFS, NHS }\end{array}$ & Pancreatic & Cohort & $\begin{array}{l}136,587 \\
14 \mathrm{HPFS}, \\
20 \mathrm{NHS}\end{array}$ & 379 & 53.7 & $F(65)$ & $\mathrm{FFQ}$ & $\begin{array}{l}\mathrm{SSB}:<143.6 \text { vs. }>11.2 \mathrm{~mL} / \text { day } \\
\mathrm{ASB}:<143.6 \text { vs. }>11.2 \mathrm{~mL} / \text { day }\end{array}$ & $\begin{array}{l}\text { RR: } 1.13(0.81-1.58) \\
\text { RR: } 1.02(0.79-1.32)\end{array}$ & $\begin{array}{l}\text { Age, sex, smoking, BMI, } \\
\text { follow-up cycle, PA, BMM, and } \\
\text { other soft drink intake. }\end{array}$ \\
\hline
\end{tabular}


Table 1. Cont.

\begin{tabular}{|c|c|c|c|c|c|c|c|c|c|c|c|}
\hline \multicolumn{12}{|c|}{ Genitourinary Cancers (Prostate, Renal Cell, Urinary Bladder, Urothelial Cell) } \\
\hline Source & Country, Study Name & Cancer Type & Study Design & $\begin{array}{c}\text { Population Follow-Up } \\
\text { (Years) }\end{array}$ & Cases & $\begin{array}{l}\text { Age (Mean/SD } \\
\text { or Range) }\end{array}$ & Sex $\%$ & $\begin{array}{l}\text { Dietary Assessment } \\
\text { Method }\end{array}$ & $\begin{array}{c}\text { Type and Amount of Beverages } \\
\text { Intake }{ }^{+}\end{array}$ & $\begin{array}{c}\text { HR/RR/OR } \\
(95 \% \mathrm{CI})\end{array}$ & Adjustments \\
\hline $\begin{array}{l}\text { Bruemmer et al, } 1997 \\
{[60]}\end{array}$ & us & Bladder & PB case-control & 620 & 215 & $45-65$ & $\mathrm{M}(62)$ & Interview & $\begin{array}{c}\text { SSB: }>240 \mathrm{vs} .<8 \mathrm{~mL} / \text { day } \\
\text { ASB: }>240<8 \mathrm{~mL} / \text { day }\end{array}$ & $\begin{array}{c}\text { OR: }: 4.4(0.2-1.1)(\mathrm{M}) \\
\text { OR } 5.7(1.2-2.69)(\mathrm{F}) \\
\text { OR: 1.6 (0.7-3.6) (M) OR: } 2.3 \\
(0.8-6.3)(\mathrm{F})\end{array}$ & Age, country, and smoking. \\
\hline $\begin{array}{l}\text { De Stefani et al., } 2007 \\
\text { [61] }\end{array}$ & Uruguay & Bladder & HB case-control & 756 & 255 & $30-89$ & $\mathrm{M}(88)$ & 64-item FFQ & $\mathrm{SB}: \geq 142 \mathrm{vs} .<142 \mathrm{~mL} /$ day & OR: $1.1(0.7-1.7)$ & $\begin{array}{l}\text { Age, sex, residence, education, } \\
\text { familiar history of UBC, BMI, } \\
\text { occupation, smoking, intake } \\
\text { of mate, coffee, tea, and milk. }\end{array}$ \\
\hline Hemelt et al., 2010 [62] & China & Bladder & HB case-control & $\begin{array}{c}792 \\
3\end{array}$ & 400 & 65.8 & $\mathrm{M}(79)$ & DQ & $\begin{array}{l}\text { SB: consumers vs. none } \\
\text { F: daily vs. none }\end{array}$ & $\begin{array}{l}\text { OR: } 2.01(1.10-3.68) \\
\text { OR: } 0.66(0.26-1.66)\end{array}$ & $\begin{array}{l}\text { Age, sex, smoking, and } \\
\text { frequency and duration of } \\
\text { smoking. }\end{array}$ \\
\hline $\begin{array}{l}\text { Radosavljevic et al., } \\
2003 \text { [63] }\end{array}$ & Serbia & Bladder & HB case-control & 260 & 130 & 64.9 & $\mathrm{M}(79)$ & 101-item FFQ & $\begin{array}{l}\text { SB: }>15.7 \mathrm{~mL} / \text { day (mean) vs. none } \\
\text { F: }>11.6 \mathrm{~mL} / \text { day (mean) vs. none }\end{array}$ & $\begin{array}{l}\text { OR: } 4.73(2.72-8.18) \\
\text { OR: } 0.30(0.18-0.50)\end{array}$ & Smoking \\
\hline Turati et al., 2015 [64] & Italy & Bladder & HB case-control & 1355 & 665 & 67 (median) & $\mathrm{M}(76)$ & DQ & $\mathrm{SB}^{2}: \geq 47 \mathrm{~mL} /$ day vs. none & OR: $1.04(0.73-1.49)$ & $\begin{array}{l}\text { Age, sex, study center, year of } \\
\text { interview, smoking, } \\
\text { education, alcohol, BMI, and } \\
\text { family history of UBC and } \\
\text { cystitis. }\end{array}$ \\
\hline Wang, 2013 [65] & us & Bladder & HB case-control & 2306 & 1007 & 64.4 & $\mathrm{M}(78)$ & $\mathrm{FFQ}$ & $\begin{array}{l}\text { SB: } \geq 255.6 \mathrm{~mL} / \text { day vs. none } \\
\text { SSB: } \leq 126 \mathrm{~mL} / \text { day vs. none } \\
\text { ASB: } \geq 309.6 \mathrm{~mL} / \text { day vs. none }\end{array}$ & $\begin{array}{l}\text { OR: } 1.34(1.05-1.70) \\
\text { OR: } 1.27(1.02-1.58) \\
\text { OR: } 1.06(0.85-1.32)\end{array}$ & $\begin{array}{l}\text { Age, sex, ethnicity, EI, and } \\
\text { smoking. }\end{array}$ \\
\hline $\begin{array}{l}\text { Chazelas et al., } 2019 \\
\text { [23] }\end{array}$ & France & Prostate & Cohort & $\begin{array}{l}101,257 \\
5.1 \text { (median) }\end{array}$ & 291 & $42.2 / 4.4$ & $\mathrm{M}(100)$ & $24 \mathrm{H}-\mathrm{DR}$ & $\begin{array}{l}\text { SFJ: }>141.7 \mathrm{vs} .<46.1 \mathrm{~mL} / \text { day (cut-off) } \\
\text { SFJ: increase by } 100 \mathrm{~mL} / \text { day } \\
\text { SSB: }>655.5 \mathrm{vs} .<14.0 \mathrm{~mL} / \text { day (cut-off) } \\
\text { SSB: increase by } 100 \mathrm{~mL} / \text { /day } \\
\text { ASB: }>7.9 \mathrm{vs}<2.7 \mathrm{~mL} / \text { day (cut-off) } \\
\text { ASB: increase by } 10 \mathrm{~mL} / \text { /day } \\
\text { FJ: }>97.8 \text { vs. }<19.9 \mathrm{~mL} / \text { day (cut-off) } \\
\text { FJ: increase by } 100 \mathrm{~mL} / \text { day }\end{array}$ & 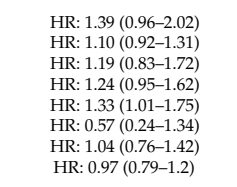 & $\begin{array}{l}\text { Smoking, education, PA, BMI, } \\
\text { and height. }\end{array}$ \\
\hline Drake et al., 2012 [35] & $\begin{array}{l}\text { Sweden, } \\
\text { MDC }\end{array}$ & Prostate & Cohort & $\begin{array}{l}8128 \\
14.9\end{array}$ & 817 & $45-73$ & $\mathrm{M}(100)$ & $\begin{array}{l}\text { 168-item FFQ, } \\
\text { 7-d menu book } \\
\text { Interview }\end{array}$ & $\begin{array}{l}\text { SSB: } 297.8 \mathrm{~mL} / \text { day (median) vs. none } \\
\text { F: } 270 \mathrm{~mL} / \text { day (median) vs. none }\end{array}$ & $\begin{array}{l}\text { HR: } 1.13(0.92-1.38) \\
\text { HR: } 0.99(0.81-1.22)\end{array}$ & $\begin{array}{l}\text { Age, year of study entry, time } \\
\text { of data collection, EI, height, } \\
\text { WC, PA, smoking, education, } \\
\text { birth in Sweden, alcohol, } \\
\text { calcium and selenium intake, } \\
\text { and risk by death from all } \\
\text { causes except PC. }\end{array}$ \\
\hline Ellison et al., 2000 [36] & $\begin{array}{l}\text { Canada, } \\
\text { NCSS }\end{array}$ & Prostate & Cohort & $\begin{array}{c}3400 \\
23\end{array}$ & 201 & $50-84$ & $\mathrm{M}(100)$ & FFQ & $\begin{array}{c}\mathrm{SB}^{2}: \geq 100 \mathrm{~mL} / \text { day vs. none } \\
\mathrm{SB}^{2}: \text { any vs. none } \\
\end{array}$ & $\begin{array}{l}\text { RR: } 1.29(0.74-2.26) \\
\text { RR:1.09 (0.78-1.35) }\end{array}$ & $\begin{array}{l}\text { Age, alcohol, smoking, BMI, } \\
\text { fiber, and EI. }\end{array}$ \\
\hline Hodge et al., 2018 [54] & Australia, MCCS & Prostate & Cohort & $\begin{array}{c}35,593 \\
19\end{array}$ & 433 & 54.6 & $\mathrm{M}(100)$ & 121-item FFQ & $\begin{array}{l}\text { SSB: } \geq 200 \mathrm{vss},<6.7 \mathrm{~mL} / \text { day } \\
\text { ASB: } \geq 200 \text { vs. }<6.7 \mathrm{~mL} / \text { day }\end{array}$ & $\begin{array}{l}\text { HR: } 1.08(0.78-1.50) \\
\text { HR: } 0.81(0.49-1.33)\end{array}$ & $\begin{array}{l}\text { Socioeconomic indexes, } \\
\text { country of birth, alcohol, } \\
\text { smoking, PA, and Med-diet } \\
\text { score. ASB also for SSB } \\
\text { consumption and WC. }\end{array}$ \\
\hline Jain et al., 1998 [66] & Canada & Prostate & PB case-control & 1253 & 617 & 69.8 & $\mathrm{M}(100)$ & Q-DH & $\mathrm{SB}^{2}:>200 \mathrm{~mL} /$ day vs. none & OR: 0.79 (0.53-1.17) & Age, EI \\
\hline $\begin{array}{l}\text { Makarem et al., } 2018 \\
{[52]}\end{array}$ & us & Prostate & Cohort & $\begin{array}{c}3184 \\
4\end{array}$ & 157 & 54.3 & $\mathrm{M}(100)$ & $\mathrm{FFQ}$ & $\begin{array}{l}\text { SFF: }>401 \mathrm{vs},<212.1 \mathrm{~mL} / \text { day (cut-off) } \\
\text { SSB: }>180 \mathrm{vs}<25.7 \mathrm{~mL} / \text { day (cut-off) } \\
\text { FJ: }>180 \mathrm{vs} .<48.9 \mathrm{~mL} / \text { day (cut-off) }\end{array}$ & $\begin{array}{l}\text { HR: } 1.06(1.03-1.09) \\
\text { HR: } 1.38(0.80-2.38) \\
\text { HR: } 1.03(1.01-1.06)\end{array}$ & $\begin{array}{l}\text { Age, smoking, BMI, EI, } \\
\text { alcohol, PA, education, WC, } \\
\text { DM, CVD, antioxidant use, } \\
\text { and energy from fat and diet } \\
\text { soda intake. }\end{array}$ \\
\hline
\end{tabular}


Table 1. Cont.

\begin{tabular}{|c|c|c|c|c|c|c|c|c|c|c|c|}
\hline Miles et al., 2018 [31] & us & Prostate & Cohort & $\stackrel{22,720}{9}$ & 1996 & $65.6(5.9)$ & $\mathrm{M}(100)$ & FFQ & $\begin{array}{l}\text { SSB: }>183 \mathrm{vs},<6 \mathrm{~mL} / \text { day (cut-off) } \\
\text { FJ: }>198 \text { vs. }<24 \mathrm{~mL} / \text { day (cut-off) }\end{array}$ & $\begin{array}{l}\text { HR: } 1.21(1.06-1.39) \\
\text { HR: } 1.07(0.094-1.22)\end{array}$ & $\begin{array}{l}\text { Age, sex, smoking, BMI, EI, } \\
\text { DM, education, race, family } \\
\text { history of PC, and PSA } \\
\text { screens. }\end{array}$ \\
\hline Sharpe et al., $2002[67]$ & Canada & Prostate & PB case-control & 875 & 399 & 61.5 & $\mathrm{M}(100)$ & $\begin{array}{c}\text { Interviews } \\
\text { or DQ }\end{array}$ & $\begin{array}{l}\mathrm{SB}^{7} \text { : daily drank vs. never drank } \\
\text { weekkly }\end{array}$ & OR: $1.0(0.7-1.4)$ & $\begin{array}{l}\text { Age, ethnicity, socioeconomic } \\
\text { status, BMI, cumulative } \\
\text { cigarette smoking, and } \\
\text { alcohol. }\end{array}$ \\
\hline Hodge et al., 2018 [54] & Australia, MCCS & Renal cell & Cohort & $\begin{array}{c}35,593 \\
19\end{array}$ & 146 & 54.6 & $M / F$ & 121-item FFQ & $\begin{array}{l}\text { SSB: } 2200 \mathrm{vs},<6.7 \mathrm{~mL} / \text { day } \\
\text { ASB: } 2200 \mathrm{vs}<<6.7 \mathrm{~mL} / \text { day }\end{array}$ & $\begin{array}{l}\text { HR: } 1.48(0.87-2.53) \\
\text { HR: } 0.92(0.46-1.84)\end{array}$ & $\begin{array}{l}\text { Socioeconomic indexes, } \\
\text { country of birth, alcohol, } \\
\text { smoking, PA, Med-diet score, } \\
\text { and sex. ASB also for SSB } \\
\text { consumption and WC }\end{array}$ \\
\hline Hu et al., 2009 [68] & Canada & Renal cell & PB case-control & 6177 & 1138 & $20-80$ & M (51) & FFQ & 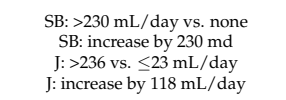 & $\begin{array}{l}\text { OR: } 1.26(0.96-1.167) \\
\text { OR } 1.05(0.07-1.13) \\
\text { OR } 1.53(1.11-199) \\
\text { OR: } 1.08(1.04-1.13)\end{array}$ & $\begin{array}{l}\text { 10-year age groups, province, } \\
\text { education, BMI, sex, EI, } \\
\text { smoking, intake of alcohol } \\
\text { meat, vegetables, and fruits. }\end{array}$ \\
\hline Lee et al., $2006[37]$ & us & Renal cell & Cohort & $\begin{array}{l}136,587 \\
14 \mathrm{HPFS} \\
20 \mathrm{NHS}\end{array}$ & 248 & 53.7 & $\mathrm{~F}(65)$ & $\mathrm{FFQ}$ & $\begin{array}{l}\text { SB: } \geq 670 \text { vs. }<47.9 \mathrm{~mL} / \text { day } \\
\text { SSB: increase by } 335 \mathrm{~mL} / \text { day } \\
\text { ASB: increase by } 335 \mathrm{~mL} / \text { day } \\
\text { FJ: increase by } 335 \mathrm{~mL} / \text { day }\end{array}$ & $\begin{array}{l}\text { RR: } 1.03(0.64-1.68) \\
\text { RR :0.95 (0.069-1.31) } \\
\text { RR: } 0.97(0.1-15) \\
\text { RR: } 1.06(0.88-1.28)\end{array}$ & $\begin{array}{l}\text { BMI, EI, alcohol, smoking, } \\
\text { history of HT, DM, } \\
\text { multivitamin use, and parity. }\end{array}$ \\
\hline $\begin{array}{l}\text { Maclure and Willet, } \\
1990[700]\end{array}$ & us & Renal cell & PB case-control & 430 & 203 & $30 \rightarrow>0$ & $\mathrm{M}(67)$ & FFQ & 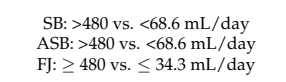 & $\begin{array}{l}\text { OR: } 2.6(1.4 .4 .8) \\
\text { OR: } 2.7(1.1-6.5) \\
\text { OR: } 0.56(0.22-1.4)\end{array}$ & $\begin{array}{l}\text { Age, sex, body weight/height, } \\
\text { EI, and education }\end{array}$ \\
\hline Ros et al., $2011[38]$ & $\begin{array}{c}10 \text { European countries } \\
\text { †, EPIC }\end{array}$ & Urothelial cell & Cohort & $\underset{9.3}{233,236}$ & 513 & $25-70$ & $\mathrm{~F}(71)$ & DQ-country specific & 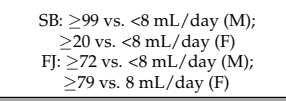 & $\begin{array}{l}\text { HR: } 1.03(0.83-1.30) \\
\text { HR: } 1.32(1.05-1.66)\end{array}$ & $\begin{array}{l}\text { Smoking, EI from fat and } \\
\text { nonfat sources. Stratified by } \\
\text { age at entry, sex, and center. }\end{array}$ \\
\hline Source & Country, Study Name & Cancer Type & Study Design & $\begin{array}{c}\text { Population Follow-Up } \\
\text { (Years) }\end{array}$ & Cases & $\begin{array}{c}\text { Age (Mean/SD } \\
\text { or Range) }\end{array}$ & Sex $(\%)$ & $\begin{array}{l}\text { Dietary Assessment } \\
\text { Method }\end{array}$ & $\begin{array}{l}\text { Type and Amount of Beverages } \\
\text { Intake }^{+}\end{array}$ & $\begin{array}{c}\mathrm{HR} / \mathrm{RR} / \mathrm{OR} \\
(95 \% \mathrm{CI})\end{array}$ & Adjustments \\
\hline Herrero et al., 1991 [71] & $\begin{array}{l}\text { Colombia, Costa Rica, } \\
\text { Mexico and Panama }\end{array}$ & Cervical & HB, PB case-control & 2033 & 622 & 46.5 & $\mathrm{~F}(100)$ & $\mathrm{FFQ}$ & FJ: $>240 \mathrm{vs},<0.8 \mathrm{~mL} / \mathrm{day}$ & OR: $0.90(0.7-1.2)$ & $\begin{array}{l}\text { Age, study site, age at 1st } \\
\text { intercourse, number of sexual } \\
\text { partners and pregnancies, } \\
\text { presence of HPV } 16 / 18, \\
\text { interval since last Pap smear, } \\
\text { and number of household } \\
\text { facilities. }\end{array}$ \\
\hline $\begin{array}{l}\text { Verreault et al. } 1989 \\
{[72]}\end{array}$ & us & Cervical & PB case-control & 416 & 189 & 20-74 & $\mathrm{F}(100)$ & 66-items FFQ & FJ: $\geq 355 \mathrm{vs.} \leq 48 \mathrm{~mL} /$ day & RR: $0.3(0.2-0.6)$ & $\begin{array}{l}\text { Age, education, smoking, } \\
\text { frequency of Pap smears, use } \\
\text { of barrier and OC, history of } \\
\text { cervical-vaginal infection, age } \\
\text { at first intercourse, and } \\
\text { number of sexual partners. }\end{array}$ \\
\hline $\begin{array}{c}\text { Inoue-Choi et al., } 2013 \\
{[39]}\end{array}$ & us & $\begin{array}{l}\text { Endometrial type I } \\
\text { Endometrial type II }\end{array}$ & Cohort & $\begin{array}{l}23,039 \\
14\end{array}$ & $\begin{array}{l}506 \\
89\end{array}$ & 61.6 & $\mathrm{~F}(100)$ & $\mathrm{FFQ}$ & 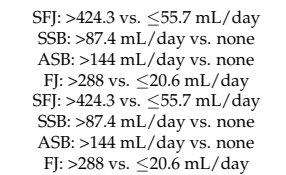 & 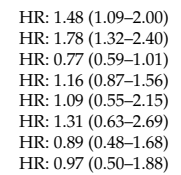 & $\begin{array}{l}\text { Age, smoking, BMI, PA, } \\
\text { alcohol, HRR, age at menarche } \\
\text { and at menopause, number of } \\
\text { live births, DM , and } \\
\text { coffee intake. }\end{array}$ \\
\hline
\end{tabular}


Table 1. Cont.

\begin{tabular}{|c|c|c|c|c|c|c|c|c|c|c|c|}
\hline Hodge et al., 2018 [54] & Australia, MCCS & $\begin{array}{c}\text { Endometrial } \\
\text { Ovarian }\end{array}$ & Cohort & $\begin{array}{c}35,593 \\
19\end{array}$ & $\begin{array}{l}167 \\
130\end{array}$ & 54.6 & $\mathrm{~F}(100)$ & 121-item FFQ & $\begin{array}{l}\text { SSB: } 2200 \mathrm{vs} .<6.7 \mathrm{~mL} / \mathrm{day} \\
\text { ASB: } 200 \mathrm{vs}<<6.7 \mathrm{~mL} \text { day } \\
\text { SSB: } 220 \mathrm{vs}<<6.7 \mathrm{~mL} / \mathrm{day} \\
\text { ASB: } \geq 200 \mathrm{vs} .<6.7 \mathrm{~mL} / \mathrm{day}\end{array}$ & $\begin{array}{l}\text { HR: } 1.02(0.54-1.91) \\
\text { HR } 0.81(0.42-.55) \\
\text { HR: } 1.35(0.71-2.56) \\
\text { HR: } 1.37(0.72-2.61)\end{array}$ & $\begin{array}{l}\text { Socioeconomic indexes, } \\
\text { country of birth, alcohol, } \\
\text { smoking, PA, Med-diet score, } \\
\text { and sex. ASB also for SSB } \\
\text { consumption and WC. }\end{array}$ \\
\hline King et al., 2013 [73] & us & Epithelial ovarian & PB case-control & $\begin{array}{c}595 \\
7\end{array}$ & 205 & $>21$ & $\mathrm{~F}(100)$ & FFQ and Interview & $\begin{array}{c}\text { SSB: } \geq 151.2 \text { vs. }<21.6 \mathrm{~mL} / 2000 \\
\text { kcal /day } \\
\text { SSB: increase by } 360 \mathrm{~mL} / \text { day }\end{array}$ & $\begin{array}{l}\text { OR: } 1.31(0.77-2.24) \\
\text { OR: } 1.63(0.94-2.83)\end{array}$ & $\begin{array}{l}\text { Age, education, race, age at } \\
\text { menarche, MS, , arity, OC use, } \\
\text { HRT, BMI, smoking, PA, DM, } \\
\text { tubal ligation, intake of fiber, } \\
\text { fat, and saturated fat. }\end{array}$ \\
\hline Leung et al., 2016 [74] & Canada & Epithelial ovarian & PB case-control & $\begin{array}{l}{ }^{2111} \\
11\end{array}$ & 524 & $40-79$ & $\mathrm{~F}(100)$ & FFQ and Interview & $\mathrm{SB}:>9.9 \mathrm{~mL} /$ day vs. none & OR: $0.97(0.72-1.31)$ & $\begin{array}{l}\text { Age, race, education, BMI, } \\
\text { smoking, alcohol, history of } \\
\text { ovarian /beast cancer OC } \\
\text { use, parity, MS , HRT, , and } \\
\text { study site. }\end{array}$ \\
\hline Song et al., 2008 [75] & US & Epithelial ovarian & PB case-control & $\begin{array}{c}2050 \\
3\end{array}$ & 781 & $35-74$ & $\mathrm{~F}(100)$ & FFQ & $\begin{array}{c}\mathrm{SB}^{3} \text { (caff) } \geq 720 \mathrm{~mL} / \text { day vs. none } \\
\mathrm{SB}^{3} \text { (not caff): } \geq 720 \mathrm{~mL} / \text { day vs. none }\end{array}$ & $\begin{array}{l}\text { OR: } 1.51(1.03-2.22) \\
\text { OR: } 2.60(1.25-5.39)\end{array}$ & $\begin{array}{l}\text { Age, BMI, education, } \\
\text { smoking, race, country, years } \\
\text { of diagnosis, number of } \\
\text { pregnancies, OC use, } \\
\text { hysterectomy, and family } \\
\text { history of } \\
\text { breast/oviarian cancer. }\end{array}$ \\
\hline & \multicolumn{11}{|c|}{ Hepatobiliary Cancers (Biliary Tract, Gallbladder, Liver) } \\
\hline Source & Country, Study Name & Cancer Type & Study Design & $\begin{array}{c}\text { Population Follow-Up } \\
\text { (Years) }\end{array}$ & Cases & $\begin{array}{c}\text { Age (Mean/SD } \\
\text { or Range) }\end{array}$ & Sex $(\%)$ & $\begin{array}{c}\text { Dietary Assessment } \\
\text { Method }\end{array}$ & $\begin{array}{c}\text { Type and Amount of Beverages } \\
\text { Intake } \\
\end{array}$ & $\begin{array}{l}\mathrm{HR} / \mathrm{RR} / \mathrm{OR} \\
(95 \% \mathrm{CI})\end{array}$ & Adjustments \\
\hline Stepien et al., $2014[28]$ & $\begin{array}{l}10 \text { European countries } \\
+, \text { EPIC }\end{array}$ & Biliary tract & Cohort & $\begin{array}{c}477,206 \\
11.4\end{array}$ & 236 & 51 & $\mathrm{~F}(70)$ & DQ-country specific & 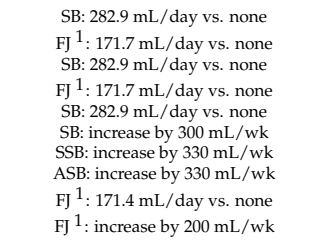 & 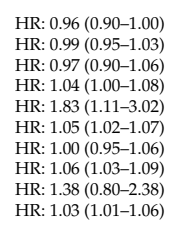 & $\begin{array}{l}\text { BMI, alcohol, EI, PA, DM, and } \\
\text { education. }\end{array}$ \\
\hline Larsson et al., 2016 [49] & $\begin{array}{l}\text { Sweden, SMC, } \\
\text { COSM }\end{array}$ & $\begin{array}{c}\text { IHBT } \\
\text { EHBT } \\
\text { Gallbladder }\end{array}$ & Cohort & $\begin{array}{c}70,832 \\
13.4\end{array}$ & $\begin{array}{l}21 \\
127 \\
71\end{array}$ & $45-83$ & $\mathrm{M}(56)$ & 96-item FFQ & $\begin{array}{l}\mathrm{SB}: \geq 400 \mathrm{~mL} / \text { day vs. none } \\
\mathrm{SB}: \geq 400 \mathrm{~mL} / \text { /day vs. none } \\
\mathrm{SB}: \geq 400 \mathrm{~mL} / \text { day vs. none }\end{array}$ & $\begin{array}{l}\text { HR: } 1.69(0.41-7.03) \\
\text { HR: } 1.79(1.02-3.13) \\
\text { HR: } 2.24(1.102-4.89)\end{array}$ & $\begin{array}{l}\text { Age, sex, education, smoking, } \\
\text { BMI, dietary protein intake, } \\
\text { and EI. }\end{array}$ \\
\hline & \multicolumn{11}{|c|}{ Hematologic Cancers (Leukemia, Lymphoma, Myeloma) } \\
\hline Source & Country, Study Name & Cancer Type & Study Design & $\begin{array}{l}\text { Population Follow-Up } \\
\text { (Years) }\end{array}$ & Cases & $\begin{array}{c}\text { Age (Mean/SD } \\
\text { or Range) }\end{array}$ & Sex $(\%)$ & $\begin{array}{l}\text { Dietary Assessment } \\
\text { Method }\end{array}$ & $\begin{array}{l}\text { Type and Amount of Beverages } \\
\text { Intake }^{+}\end{array}$ & $\begin{array}{c}\mathrm{HR} / \mathrm{RR} / \mathrm{OR} \\
(95 \% \mathrm{CI})\end{array}$ & Adjustments \\
\hline $\begin{array}{l}\text { Schernhammer et al., } \\
2012[24]\end{array}$ & $\begin{array}{l}\text { US, } \\
\text { HPFS, NHS }\end{array}$ & $\begin{array}{c}\text { Leukemia } \\
\text { Multiple myeloma } \\
\text { NHL }\end{array}$ & Cohort & $\begin{array}{l}136,587 \\
14 \mathrm{HPFS} \\
20 \mathrm{NHS}\end{array}$ & $\begin{array}{l}339 \\
285 \\
1324\end{array}$ & 53.7 & $\mathrm{~F}(65)$ & FFQ & 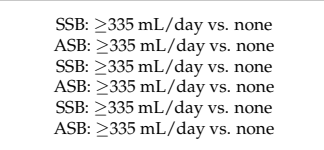 & $\begin{array}{l}\text { RR: } 1.06(0.56-2.00) \\
\text { RR: } 1.42(1.00-2.02) \\
\text { RR } 1.47(7.076-2.83) \\
\text { RR } 1.29(0.89-1.89) \\
\text { RR: } 1.34(0.998-1.83) \\
\text { RR: } 1.13(0.94-1.34)\end{array}$ & $\begin{array}{l}\text { Age, , BII, EI, PA, alcohol, } \\
\text { race, fruit and vegetableses } \\
\text { consumption, menopause, } \\
\text { and HT. SSB were adjusted } \\
\text { for use of ASB and vice-versa. }\end{array}$ \\
\hline $\begin{array}{l}\text { McCullough et al., } 2014 \\
\text { [40] }\end{array}$ & $\begin{array}{l}\text { US, } \\
\text { CPS-II NCH }\end{array}$ & NHL & Cohort & $\begin{array}{c}100,442 \\
10\end{array}$ & 1196 & $47-95$ & $\mathrm{~F}(57)$ & Willett FFQ & $\begin{array}{l}\text { ASB: }>355 \mathrm{~mL} / \text { day vs. none } \\
\text { SSB: }>355 \mathrm{~mL} / \text { day vs. none }\end{array}$ & $\begin{array}{l}\text { RR: }: 0.92(0.73-1.17) \\
\text { RR: } 1.10(0.77-1.58)\end{array}$ & 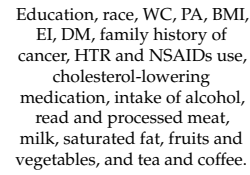 \\
\hline
\end{tabular}


Table 1. Cont.

\begin{tabular}{|c|c|c|c|c|c|c|c|c|c|c|c|}
\hline & \multicolumn{11}{|c|}{ Upper Aerodigestive Cancers (Larynx, Oral Cavity, Oropharyngeal Squamous Cell, Pharynx) } \\
\hline Source & Country, Study Name & Cancer Type & Study Design & $\begin{array}{l}\text { Population Follow-Up } \\
\text { (Years) }\end{array}$ & Cases & $\begin{array}{c}\text { Age (Mean/SD } \\
\text { or Range) }\end{array}$ & Sex $(\%)$ & $\begin{array}{l}\text { Dietary Assessment } \\
\text { Method }\end{array}$ & $\begin{array}{c}\text { Type and Amount of Beverages } \\
\text { Intake }{ }^{+}\end{array}$ & $\begin{array}{c}\mathrm{HR} / \mathrm{RR} / \mathrm{OR} \\
(95 \% \mathrm{CI})\end{array}$ & Adjustments \\
\hline Zvrko et al, 2008 [82] & Montenegro & Larynx & HB case-control & $\begin{array}{c}216 \\
2\end{array}$ & 108 & $59.9(9.7)$ & $\mathrm{M}(82)$ & DQ & SB: yes vs. no & OR: $0.38(0.16-0.92)$ & $\begin{array}{l}\text { Age, sex, smoking, alcohol, } \\
\text { coffee, diet, personal and } \\
\text { familiar medical history, } \\
\text { eduction, housing and work } \\
\text { conditions, and exposure to } \\
\text { toxic components. }\end{array}$ \\
\hline Ren et al., $2010[34]$ & $\begin{array}{l}\text { US, } \\
\text { NIH-AARP-DHS }\end{array}$ & $\begin{array}{c}\text { Larynx } \\
\text { Pharynx } \\
\text { Oral cavity }\end{array}$ & Cohort & $\underset{2}{481,563}$ & $\begin{array}{l}307 \\
178 \\
391\end{array}$ & $50-71$ & $\mathrm{M}(59)$ & 124-item FFQ & $\begin{array}{l}\text { SB: } \geq 355 \mathrm{vs} . \leq 355 \mathrm{~mL} / \text { day } \\
\text { SB: } \geq 355 \mathrm{vs} . \leq 355 \mathrm{~mL} / \mathrm{day} \\
\text { SB: } \geq 355 \mathrm{vs} . \leq 355 \mathrm{~mL} / \mathrm{day}\end{array}$ & $\begin{array}{l}\text { HR: } 0.82(0.55-1.23) \\
\text { HR: } 0.76(0.46-1.25) \\
\text { HR: } 0.77(0.54-1.09)\end{array}$ & $\begin{array}{l}\text { Age, sex, smoking, alcohol } \\
\text { drinking, BMI, EI, education, } \\
\text { ethnicity, PA, intake of fruit, } \\
\text { vegetables, and red and } \\
\text { white meat. }\end{array}$ \\
\hline \multirow[t]{2}{*}{ Kreimer et al., 2006 [84] } & $\begin{array}{l}9 \text { countries } \neq \text {, } \\
\text { IARC-MOCS }\end{array}$ & oosc & HB case-control & 3402 & 1670 & NR & $\mathrm{M} / \mathrm{F}$ & FFQ & F: height vs. low intake & OR: $0.8(0.6-1.1)$ & $\begin{array}{l}\text { Age, sex, country, education, } \\
\text { BMI, smoking, chewing, and } \\
\text { alcohol. }\end{array}$ \\
\hline & \multicolumn{11}{|c|}{ Other Cancers } \\
\hline Source & Country, Study Name & Cancer Type & Study Design & $\begin{array}{c}\text { Population Follow-Up } \\
\text { (Years) }\end{array}$ & Cases & $\begin{array}{c}\text { Age (Mean/SD } \\
\text { or Range) }\end{array}$ & Sex $(\%)$ & $\begin{array}{l}\text { Dietary Assessment } \\
\text { Method }\end{array}$ & $\underset{\mathrm{Nype} \text { and Amount of Beverages Intake }}{+}$ & $\begin{array}{c}\text { HR/RR/OR } \\
(95 \% \mathrm{CI})\end{array}$ & Adjustments \\
\hline Vincenti et al., 2008 [85] & Italy & $\begin{array}{l}\text { Cutaneous } \\
\text { melanoma }\end{array}$ & PB case-control & 118 & 59 & 56 & $\mathrm{~F}(53)$ & 188-item FFQ & $\begin{array}{l}\text { FJ (no OJ): increase by } 10 \mathrm{~mL} / \text { day } \\
\text { OJ: increase by } 10 \mathrm{~mL} / \text { day }\end{array}$ & $\begin{array}{l}\text { RR: } 0.95(0.87-1.03) \\
\text { RR: } 0.94(0.88-1.00)\end{array}$ & $\begin{array}{c}\text { EI, family history of } \\
\text { melanoma, skin type, history } \\
\text { of sunlight exposure, and } \\
\text { sunburns. }\end{array}$ \\
\hline Dubrow et al., $2012[47]$ & us & Glioma & Cohort & $\begin{array}{c}545,771 \\
10\end{array}$ & 904 & 62.8 (median) & $\mathrm{M}(60)$ & FFQ & $\mathrm{SB}:>720 \mathrm{~mL} /$ day vs. none & HR: $0.87(0.65-1.15)$ & $\begin{array}{l}\text { Age, sex, race, EI, height, fruit } \\
\text { and vegetables intake, and } \\
\text { nitrite intake from plants }\end{array}$ \\
\hline Luqman et al., 2014 [86] & Pakistan & Lung & HB case-control & 1200 & 400 & $<40>70$ & $\mathrm{M}(73)$ & $\mathrm{DQ}$ & J: yes vs. no & OR: $0.3(0.3-0.4)$ & Not reported \\
\hline Wu A. et al., $1997[87]$ & us & $\begin{array}{c}\text { Small } \\
\text { intestine }\end{array}$ & PB case-control & 1034 & 36 & $30-65$ & $\mathrm{M}(69)$ & Interview & $\mathrm{SSB}^{7}$ : daily vs. never & OR: $3.6(1.3-9.8)$ & Age, ethnicity, and sex. \\
\hline $\begin{array}{c}\text { Zamora-Ros et al., } 2018 \\
{[48]}\end{array}$ & $\begin{array}{c}10 \text { European countries } \\
+, \text { EPIC }\end{array}$ & Thyroid & Cohort & $\begin{array}{c}477,206 \\
11.4\end{array}$ & 748 & 51 & $\mathrm{~F}(70)$ & DQ- country specific & $\begin{array}{c}\mathrm{FJ}^{1}:>94 \mathrm{vs},<1 \mathrm{~mL} / \text { day } \\
\mathrm{FJ}^{1}: \text { increase }<5 y 0 \mathrm{~mL} / \text { day }\end{array}$ & $\begin{array}{l}\text { HR: } 1.23(0.98-1.53) \\
\text { HR: } 1.02(0.99-1.06)\end{array}$ & $\begin{array}{l}\text { Age, sex, smoking status, BMI, } \\
\text { EI, alcohol, PA, education, } \\
\text { center, menopausal status and } \\
\text { type, OC use, and infertility } \\
\text { problems. }\end{array}$ \\
\hline \multicolumn{12}{|c|}{ Overall Cancers } \\
\hline Source & Country, Study Name & Cancer Type & Study Design & $\begin{array}{l}\text { Population Follow-Up } \\
\text { (Years) }\end{array}$ & Cases & $\begin{array}{l}\text { Age (Mean/SD } \\
\text { or Range) }\end{array}$ & Sex $(\%)$ & $\begin{array}{l}\text { Dietary Assessment } \\
\text { Method }\end{array}$ & Type and Amount of Beverages Intake & $\begin{array}{l}\mathrm{HR} / \mathrm{RR} / \mathrm{OR} \\
(95 \% \mathrm{CI})\end{array}$ & Adjustments \\
\hline Bassett et al., $2020[50]$ & Australia, MCCS & $\begin{array}{l}\text { Non-obesity } \\
\text { related }{ }^{*}\end{array}$ & Cohort & $\begin{array}{c}35,109 \\
19\end{array}$ & 4789 & $27-76$ & $\mathrm{~F}(61)$ & 121-item FFQ & $\begin{array}{l}\text { SSB: }>375 \mathrm{vs} \text {. none or }<12.5 \mathrm{~mL} / \text { day } \\
\text { ASB: }>375 \mathrm{vs} \text {. none or }<12.5 \mathrm{~mL} / \text { day }\end{array}$ & $\begin{array}{l}\text { HR: } 1.02(0.86-1.21) \\
\text { HR: } 1.23(1.02-1.48)\end{array}$ & $\begin{array}{l}\text { Alcohol, country of birth, } \\
\text { Med-diet score, , PA, } \\
\text { socio-economic position, sex, } \\
\text { and smoking. ASB also } \\
\text { adjusted for SSB intake. }\end{array}$ \\
\hline
\end{tabular}


Table 1. Cont

\begin{tabular}{|c|c|c|c|c|c|c|c|c|c|c|c|}
\hline $\begin{array}{l}\text { Makarem et al., } 2018 \\
{[52]}\end{array}$ & us & $\begin{array}{l}\text { Breast, Colorectal, } \\
\text { Prostate }\end{array}$ & Cohort & $\underset{4}{3184}$ & 565 & 54.3 & $\mathrm{~F}(53)$ & $\mathrm{FFQ}$ & $\begin{array}{c}\text { SFJ: }>501 \text { vs. }<73.2 \mathrm{~mL} / \text { day } \\
\text { SSBB }>>180 \mathrm{~mL} / \text { day vs. none } \\
\text { FJ: }>216 \text { vs. }<23 \mathrm{~mL} / \text { day (cut-off) }\end{array}$ & $\begin{array}{l}\text { HR: } 1.28(0.97-1.70) \\
\text { HR: } 1.000(0.79-1.27) \\
\text { HR: } 1.05(0.80-1.38)\end{array}$ & $\begin{array}{l}\text { Age, sex, EI, alcohol, smoking, } \\
\text { and BMI. }\end{array}$ \\
\hline Hodge et al., 2018 [54] & Australia, MCCS & Obesity-related & Cohort & $\begin{array}{c}35,593 \\
19\end{array}$ & 3283 & 54.6 & $\mathrm{~F}(100)$ & 121-item FFQ & $\begin{array}{l}\text { SSB: }: 200 \mathrm{vs} .<6.7 \mathrm{~mL} / \text { day } \\
\text { ASB: } \geq 200 \text { vs. }<6.7 \mathrm{~mL} / \text { day }\end{array}$ & $\begin{array}{l}\text { HR: } 1.14(0.93-1.39) \\
\text { HR: 1.00 (0.79-1.27) }\end{array}$ & 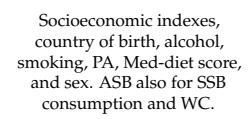 \\
\hline $\begin{array}{l}\text { Chazelas et al., } 2019 \\
{[23]}\end{array}$ & $\begin{array}{l}\text { France, } \\
\text { NNS }\end{array}$ & $\begin{array}{l}\text { Breast, Colorectal, } \\
\text { Prostate }\end{array}$ & Cohort & $\begin{array}{l}101,257 \\
5.1 \text { (median) }\end{array}$ & 2193 & $42.2 / 14.4$ & $\mathrm{~F}(78)$ & $24 \mathrm{H}-\mathrm{DR}$ & 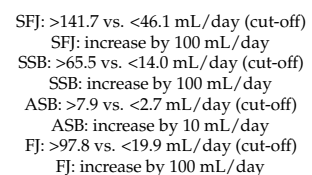 & 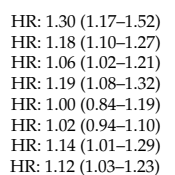 & $\begin{array}{l}\text { Smoking, education, PA, BMI, } \\
\text { and height. }\end{array}$ \\
\hline
\end{tabular}

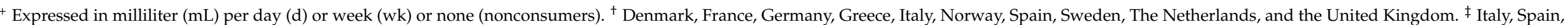

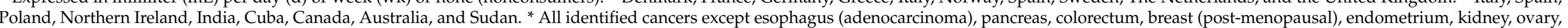

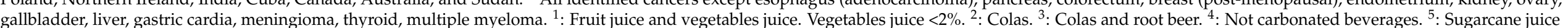

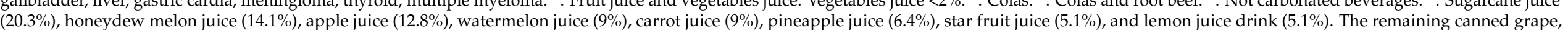

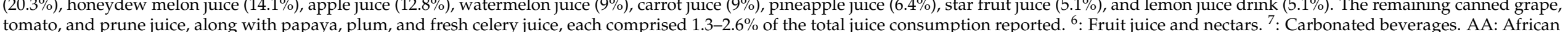

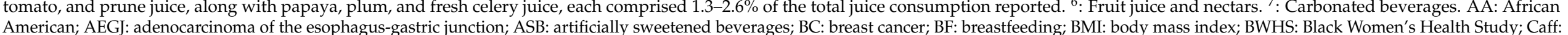

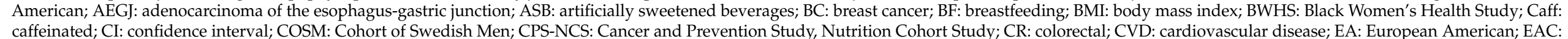

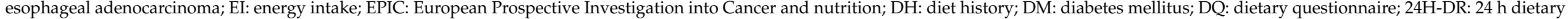

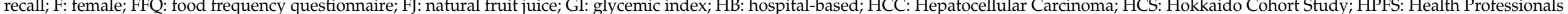

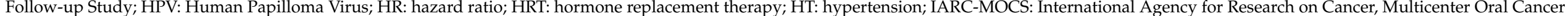

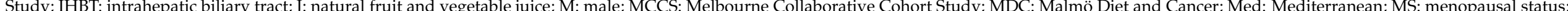

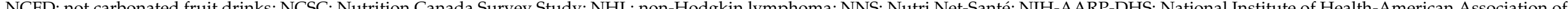

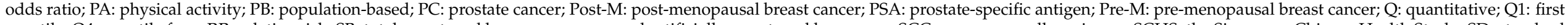

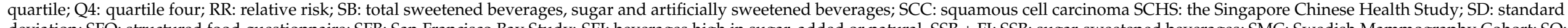

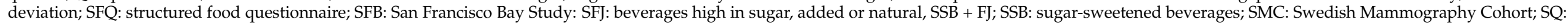

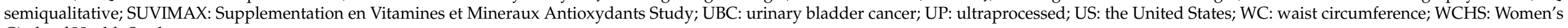
Circle of Health Study. 
Table 2. Summary of the results of the meta-analysis (random effects model).

\begin{tabular}{|c|c|c|c|c|c|c|c|c|}
\hline \multirow{2}{*}{ Cancer Type } & \multirow{2}{*}{ Exposure } & \multicolumn{2}{|c|}{$\mathbf{N}^{\circ}$ of Studies } & \multirow{2}{*}{ RR $(95 \%$ CI) } & \multirow{2}{*}{$I^{2}(\%)$} & \multirow{2}{*}{$\mathrm{Tau}^{2}$} & \multirow{2}{*}{$\begin{array}{l}p \text { within } \\
\text { Group }^{+}\end{array}$} & \multirow{2}{*}{$95 \%$ PI } \\
\hline & & Cohort & Case-Control & & & & & \\
\hline Breast & SSB & 4 & 3 & $\begin{array}{c}1.14 \\
(1.01-1.30)\end{array}$ & 0.0 & 0.0073 & 0.69 & $0.88,1.47$ \\
\hline Breast & FJ & 3 & 0 & $\begin{array}{c}1.13 \\
(0.93-1.38)\end{array}$ & 0.0 & 0.0017 & 0.79 & $0.52,2.46$ \\
\hline Breast Pre-M & SSB & 3 & 2 & $\begin{array}{c}1.37 \\
(0.99-1.88)\end{array}$ & 55.7 & 0.0358 & 0.06 & $0.68,2.76$ \\
\hline Breast Post-M & SSB & 4 & 2 & $\begin{array}{c}1.18 \\
(0.79-1.75)\end{array}$ & 54.8 & 0.1080 & 0.05 & $0.43,3.23$ \\
\hline Colorectal & SSB & 4 & 0 & $\begin{array}{c}1.18 \\
(0.99-1.41)\end{array}$ & 0.0 & 0.0039 & 0.71 & $0.82,1.69$ \\
\hline Colorectal & FJ & 2 & 2 & $\begin{array}{c}0.79 \\
(0.16-3.87)\end{array}$ & 88.5 & 0.8629 & $<0.001$ & $0.008,73.94$ \\
\hline Colorectal * & FJ & 2 & 1 & $\begin{array}{c}1.29 \\
(0.78-2.12)\end{array}$ & 0.0 & 0.0120 & 0.63 & $0.17,9.81$ \\
\hline Colorectal & SB & 0 & 3 & $\begin{array}{c}2.02 \\
(0.45-9.01)\end{array}$ & 62.9 & 0.2711 & 0.07 & $0.00,5753.1$ \\
\hline Colorectal * & SB & 0 & 2 & $\begin{array}{c}1.57 \\
(0.74-3.35)\end{array}$ & 0.0 & 0.0010 & 0.67 & - \\
\hline Bladder & SB & 0 & 5 & $\begin{array}{c}1.66 \\
(0.78-3.56)\end{array}$ & 83.4 & 0.3226 & $<0.001$ & $0.22,12.37$ \\
\hline Bladder * & SB & 0 & 4 & $\begin{array}{c}1.27 \\
(0.85-1.90)\end{array}$ & 25.3 & 0.0425 & 0.26 & $0.45,3.60$ \\
\hline Prostate & SSB & 5 & 0 & $\begin{array}{c}1.18 \\
(1.10-1.27)\end{array}$ & 0.0 & 0.0012 & 0.92 & $1.03,1.35$ \\
\hline Prostate & FJ & 4 & 0 & $\begin{array}{c}1.03 \\
(1.01-1.05)\end{array}$ & 0.0 & 0.0001 & 0.93 & $0.98,1.09$ \\
\hline Prostate & SB & 1 & 2 & $\begin{array}{c}0.97 \\
(0.56-1.69)\end{array}$ & 2.9 & 0.0241 & 0.36 & $0.07,12.7$ \\
\hline Renal cell & SB & 1 & 2 & $\begin{array}{c}1.44 \\
(0.46-4.50)\end{array}$ & 65.4 & 0.1559 & 0.056 & $0.00,604.16$ \\
\hline Pancreatic & SB & 4 & 4 & $\begin{array}{c}1.28 \\
(0.95-1.72)\end{array}$ & 58.6 & 0.0962 & 0.02 & $0.56,2.90$ \\
\hline Pancreatic & SSB & 4 & 2 & $\begin{array}{c}1.01 \\
(0.92-1.11)\end{array}$ & 0.0 & 0.0016 & 0.92 & $0.87,1.17$ \\
\hline Pancreatic & ASB & 3 & 2 & $\begin{array}{c}1.07 \\
(0.77-1.48)\end{array}$ & 43.6 & 0.0480 & 0.13 & $0.48,2.36$ \\
\hline
\end{tabular}

* Results excluding outliers; ${ }^{+} p$ values of Cochran's Q-test heterogeneity. ASB: artificial sweetened beverage; FJ: fruit juice; PI: prediction intervals; Post-M: post-menopausal; Pre-M: pre-menopausal; RR: risk ratio; CI: confidence interval; SB: sweetened beverage (including both SSBs and ASBs); SSB: sugar-sweetened beverage.

\subsection{Sweet Beverages and Risk of Breast Cancer}

Nine publications reported data on breast cancer, four case-control $[57,58,69,80]$ and five cohort studies $[23,51-53,55]$. In the meta-analysis with six publications, including four cohort studies $[23,52,53,55]$ and two case-controls [57,80], a significant positive association between high SSB consumption and breast cancer risk was observed (RR: 1.14, 95\% CI: 1.0-1.3) (Table 2). No associations were found for FJ intake (Table 2). Marzbani et al. [58] reported a positive association with SBs (OR: 2.8, 95\% CI: 1.9-4.3), but no associations were found for ASBs. Subgroup analyses for SSB consumption did not explain further heterogeneity (Table S1).

\subsubsection{Sweet Beverages and Risk of Pre-Menopausal Breast Cancer}

Three cohort publications $[23,53,55]$ and one case-control (taken as two as indices were separated by ethnicity) [57] were included in the analysis of SSB intake and premenopausal breast cancer. Their pooled analysis showed a borderline statistically nonsignificant positive association (RR: 1.37, 95\% CI: 0.99-1.88) (Figure S1), which reached the significance in the subgroup analysis including only cohort studies (RR: 1.60, 95\% CI: 1.08-2.37) (Table S1). A cohort study from 2019 [23] also reported data for ASB, FJ and SFJ intake and only indicated a positive association for SFJs (HR: 1.28, 95\% CI: 1.09-1.83). 


\subsubsection{Sweet Beverages and Risk of Post-Menopausal Breast Cancer}

A meta-analysis of four cohort studies [23,53-55] and one case-control (taken as two as indices were separated by ethnicity) [57] of SSBs showed non-significant results (Table 2). We performed subgroup analyses based on study design, country, and beverage intake categories. No statistically significant results were found from the heterogeneity test between groups (Table S1). Chazelas et al. [23] investigated the relationship with SFJ consumption and observed a positive association (HR: 1.44, 95\% CI: 1.05-1.99). No significant results were reported for ASBs.

\subsection{Sweet Beverages and Risk of Intestinal and Colorectal Cancer}

Eight publications reported data on colorectal cancer, four case control [88-91] and four cohort studies $[23,52,54,56]$. A borderline positive association was observed with SSB intake using the random-effect model (RR: 1.18, 95\% CI: 0.99-1.41) (Figure S1). No significant results were found either for SBs or for FJs (RR: 2.02, 95\% CI: $0.45-9.01$ (SB); RR: 0.79, 95\% CI: 0.16-3.87 (FJ) (Figure S2). After the exclusion of one outlier, results for the random-effect model remained non-significant. No associations were found for colorectal cancer risk and ASBs. With regard to rectal cancer, no associations were observed with ASBs, SSBs or fruit and vegetables juices [92]. A case-control study on small intestine cancer [65] indicated a significant positive association with SSB consumption (OR: 3.6, 95\% CI: $1.3-9.8)$.

\subsection{Sweet Beverages and Risk of Esophageal Cancer}

Three publications, one cohort [34] and two case-control studies [59,93] reported data on different types of esophageal cancers, including esophagus-gastric junction, esophageal adenocarcinoma and squamous cell carcinoma. No significant associations were shown between SB, SSB and ASB consumption and esophageal cancers risk.

\subsection{Sweet Beverages and Risk of Gastric Cancer}

One case-control [59] and two cohort studies [34,54] reported data on different types of gastric cancer (overall, cardia and non-cardia) and SBs, ASBs or SSBs showing no significant associations.

\subsection{Sweet Beverages and Risk of Pancreatic Cancer}

Eleven publications, six cohort [41-45] and five case-control studies [76-79,81] reported data on pancreatic cancer. No significant results were observed for SBs, SSBs or ASBs (Table 2). Although high heterogeneity was observed for $\mathrm{SBs}\left(\mathrm{I}^{2}=58.6, p=0.02\right)$ and ASBs $\left(\mathrm{I}^{2}=43.6, p=0.13\right.$ ) (Table 2$)$, after performing subgroup analyses results slightly improved but remained non-significant (Table S1). No association was observed between FJ intake and pancreatic cancer risk.

\subsection{Sweet Beverages and Risk of Genitourinary Cancer \\ 3.7.1. Bladder}

Six case-control studies [60-65] reported data on bladder cancer. No association between SB consumption and bladder cancer risk was observed in the random-effect meta-analysis including five case-control studies [61-65] (Figure S2). We observed a high heterogeneity in the meta-analysis $\left(\mathrm{I}^{2}=83.4 \%, p=0.0001\right)$. Although heterogeneity was reduced after excluding outliers and doing subgroup analyses, the associations were positive but non-significant (Table S1). A US study suggested a statistically significant relation between SB intake and bladder cancer risk [65]. Two case-control studies [60,65] also considered SSBs and ASBs separately. In a Chinese case-control study [62], SSB intake was suggested as a risk factor for bladder cancer, although no association was found for FJs. Similarly, in a Serbian study [63], no significant association was observed between FJs and bladder cancer risk. 


\subsubsection{Prostate}

Eight publications, six cohorts $[23,31,35,36,52,54]$ and two case-controls [66,67] showed data on prostate cancer. No significant associations were reported for SBs from quantitative analysis. However, positive relations were observed in the random-effect model for SSBs (RR: $1.18,95 \%$ CI: $1.10-1.27$ ) and FJs (RR: $1.03,95 \%$ CI: $1.01-1.05)$. The results remained the same in a subgroup analysis with 3 non-US (France, Spain, Australia) studies (RR: 1.13, 95\% CI: 1.03-1.24) (Table S1). Two cohorts [23,54] reported data on ASB intake and only one [23] found an increased prostate cancer risk of 33\% (HR: 1.33, 95\% CI: 1.01-1.75).

\subsubsection{Renal and Urothelial Cell Cancer}

Four publications, two case control [68,70] and two cohort studies [37,54] provided data on renal cell cancer. For our meta-analysis, we selected three publications, two case-control [68,70] and one control study [37] on SBs, but the random-effect meta-analysis showed non-significant results (Table 2). Despite observing a high heterogeneity $\left(\mathrm{I}^{2}=65.4 \%, p\right.$-value $\left.=0.058\right)$, no outliers were found, and the number of studies was too low to perform subgroup analyses $(\mathrm{n}=3)$. One case control study [70] reported a positive association with the intake of ASBs (OR: 2.7, 95\% CI: 1.1-6.5) but not the other two [37,54]. No significant results were reported for SSBs or FJs, despite one case-control [68] finding a positive association with the consumption of fruit and vegetable juices taken together (OR: 1.53, 95\% CI: 1.18-1.99). The EPIC cohort study [38] reported data on urothelial cell cancer and its association with SBs and FJs. A significant positive association was found only with FJ intake (HR: 1.32, 95\% CI: 1.05-1.66).

\subsection{Sweet Beverages and Risk of Gynecological Cancers}

Two case-control studies [71,72] investigated the relationship between FJ intake and cervical cancer risk. Only one of them [72] found an inverse association (RR: 0.3, 95\% CI: $0.2-0.6)$. Two cohort studies $[39,54]$ reported data on different types of beverages (SSBs, ASBs, FJs and SFJs) and endometrial cancer risk. Only one of them [39] found significant positive associations with both SSBs (HR: 1.78, 95\% CI: 1.32-2.40) and SFJs (HR: 1.48, 95\% CI: 1.09-2.00). Finally, three case-control studies [69-71] reported data on epithelial ovarian cancer risk. Only one of them [71] found positive associations for caffeinated (OR: 1.51, 95\% CI: 1.03-2.22) and non-caffeinated SBs (OR: 2.60, 95\% CI: 1.25-5.36). No significant associations were reported for ovarian cancer risk [50].

\subsection{Sweet Beverages and Risk of Hepatobiliary Cancers}

Two cohort studies $[28,49]$ reported data on different types of sweet beverages and various types of hepatobiliary cancers. The EPIC cohort [28] found no significant results regarding the consumption of either SBs or FJs and biliary tract cancer risk. However, a positive association was observed between both SBs (HR: 1.89, 95\% CI: 1.11-3.02) and FJs (RR: 1.03, 95\% CI: 1.01-1.06) and hepatocellular carcinoma risk. The Swedish Mammography Cohort and the Cohort of Swedish Men [49] found significant positive associations with both gallbladder (HR: 2.24, 95\% CI: 1.02-4.89) and extrahepatic biliary tract cancer risks (HR: 1.79, 95\% CI: 1.02-3.13). No significant results were reported for intrahepatic biliary tract cancer risk.

\subsection{Sweet Beverages and Risk of Hematologic Cancers}

One cohort study [24] reported data on leukemia and multiple myeloma and its association with SSB and ASB intake. Significant associations were found between the consumption of ASBs and leukemia risk (RR: 1.42, 95\% CI: 1.00-2.02). No associations were observed in two cohorts $[24,40]$ as regards SSBs or ASBs and non-Hodgkin lymphoma risk. 


\subsection{Sweet Beverages and Risk of Upper Aerodigestive Cancers}

Four studies [34,82-84] reported data on upper aerodigestive cancers. One US-based cohort [34] showed no significant association between SB intake and pharyngeal, laryngeal and oral cavity cancer risks. A case-control study from Montenegro [82] suggested an inverse relation between SBs and larynx cancer risk. The consumption of FJs was inversely associated with oral cavity cancer risk in one case-control study [83] though not in another [84].

\subsection{Sweet Beverages and Risk of Other Cancers}

Single studies reported data on different types of cancer and their link with sweet beverages. No significant associations were reported for cutaneous melanoma [85], glioma [47] or thyroid cancer risk [48] and any type of sweetened beverages. One case-control study [86] reported an inverse association between natural juices (fruit and vegetables) and lung cancer risk (OR: 0.3, 95\% CI: 0.3-0.4).

\subsection{Sweet Beverages and Risk of Overall Cancer}

An Australian cohort [50] investigated the association between SSBs and ASBs and the risk of non-obesity-related cancers; they reported a positive association only with ASBs (HR: 1.23, 95\% CI: 1.02-1.48). Two cohorts [23,52] assessed the relationships between the intake of several types of sweet beverages and obesity-related cancer risk. Only one of them [23] showed positive associations with SSBs (HR: 1.06; 95\% CI: 1.02-1.21), FJs (HR: 1.14, 95\% CI: $1.01-1.29$ ) and SFJs (HR: 1.30, 95\% CI: 1.17-1.52). No association was found for ASBs and obesity-related cancer risk.

\subsection{Quality of Included Studies}

According to the ROBINS-E tool (Figure 2a, Table S2), 13 of 27 cohort studies presented a moderate overall risk of bias. This is due to some bias being detected mostly in the classification of the exposure domain, deviation from the intended intervention and missing data. Missing data bias was not evaluated for 5 cohorts [36,39,43,51,52], as the publications did not report enough information. All studies fulfilled the criteria of low risk of bias for selection of participants' domain. In addition, $3[36,37,54]$ of 27 studies did not adjust the statistical analysis for all potential confounders. Therefore, they were classified at moderate risk of bias. Only one study [50] was classified as moderate risk of bias for outcome measurement, and another [56] for the selection of reported outcomes.

According to the NOS (Figure 2b, Table S3) most of the case-control studies (29 of 37) presented a moderate overall risk of bias; 7 publications presented a serious risk, whereas 1 indicated a low risk. The risk of bias due to the selection of the groups was classified as moderate for 35 studies, high for $2[58,82]$ and low for another $2[59,66]$. Most of the case-control studies adjusted their results for relevant and additional confounders and were classified as moderate or low risk of bias for comparability between groups. In addition, 5 were considered as serious risk for this domain, because 4 of them did not adjust for all important confounders $[60,63,66,92]$ and 1 [79] reported results from an unadjusted analysis. Moreover, 5 studies $[81,86,88,89]$ did not report this information and were classified as 'no information' category. The risk of bias due to ascertainment of the exposure was considered moderate in all case-control studies. 


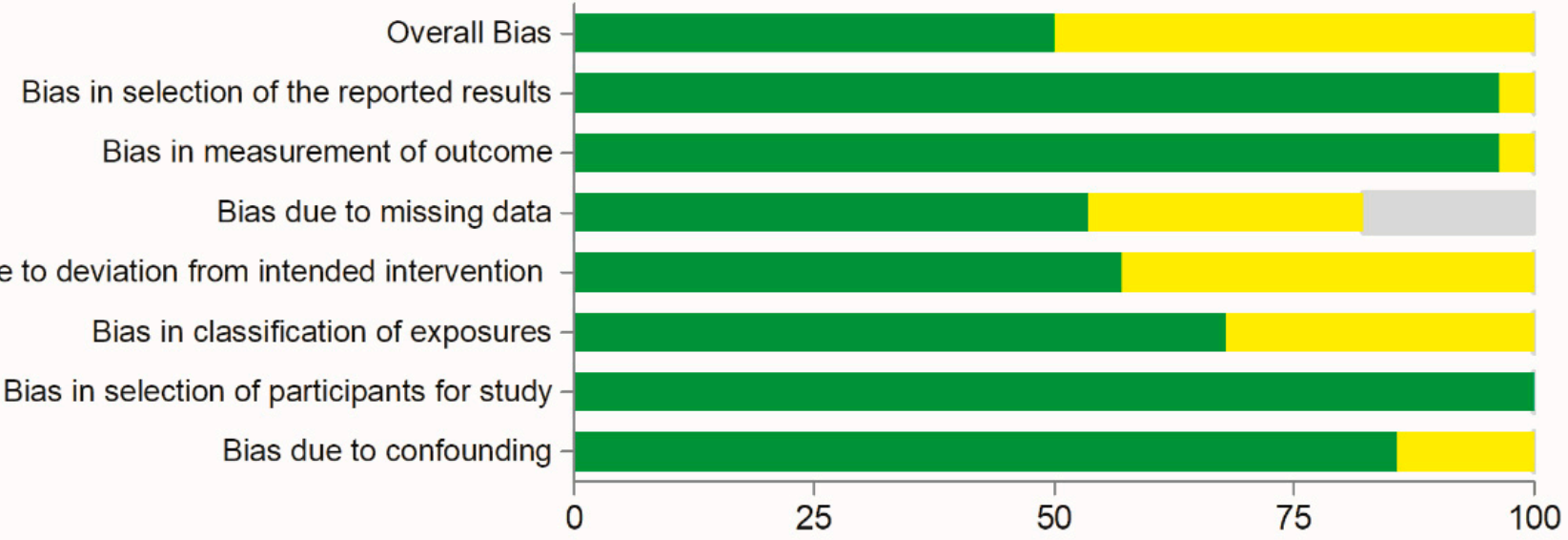

(a) Proportion fulfilled (\%)

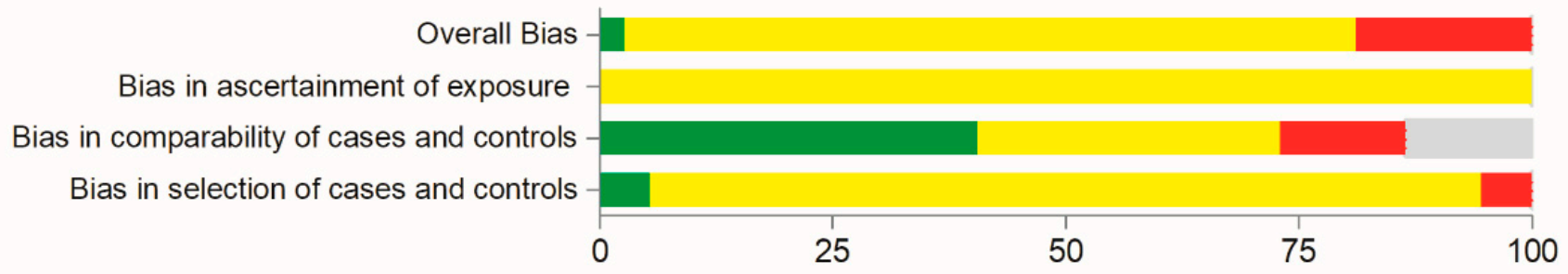

(b) Proportion fulfilled (\%)

\section{Serious risk Moderate risk Low risk No information}

Figure 2. Risk of bias in the included studies. Legend: (a) risk of bias in cohort studies according to the Risk of Bias in Non-randomized Studies - of Exposures (ROBINS-E) tool and (b) risk of bias in case-control studies according to the Newcastle-Ottawa Scale (NOS).

\section{Discussion}

\subsection{Association between Consumption of Sweet Beverages and Cancer Risk}

The aim of this study was to assess the relationships between different groups of sweet beverages and site-specific or overall cancer risk. We conducted a meta-analysis when at least three studies reported data for the same exposure (sweet beverage type) and outcome (cancer site). We found several statistically significant and borderline positive associations between the consumption of SBs, especially SSBs, and in some cases ASBs or FJs, and several cancer risks.

Regarding breast cancer, the meta-analysis showed a positive association using random effects, with a 14\% higher risk for SSBs, but non-statistically significant results for pre- and post-menopausal breast cancer. However, after performing subgroup analyses by study type, cohort studies showed significant positive results for pre-menopausal breast cancer and SSBs. Chazelas et al. [23] reported a positive linear trend between SSB intake and breast/pre-menopausal breast cancer risk when SSB consumption increased by $100 \mathrm{~mL} /$ day. In line with our results, current evidence supports the World Cancer Research Fund/American Institute for Cancer Research (WCRF/AICR) recommendations of reducing or avoiding SSB intake for breast cancer prevention [94]. One US case-control study [57] conducted a separate analysis for African-American and European-American women. This showed a positive link between SSB intake and post-menopausal breast cancer risk for European-American women only. Likewise, two other cohorts that included mostly Caucasian women $[53,54]$ showed similar results. This evidence suggests that ethnic differences may play a role. However, we could not explore this association as no other studies included women of African descent. In fact, evidence on the role of nutritional factors in breast cancer for this population is limited and inconclusive [95]. Our 
meta-analysis did not find significant associations between FJs and breast cancer risk. With regards to the SFJ group, comparing highest versus lowest consumption, Chazelas et al. [23] reported positive relations for SFJs and total, pre- and post-menopausal breast cancer risk. Conversely, Makarem et al. [52] showed no significant associations. A publication from the US [69] found no positive associations for SBs and breast cancer risk; however, a recent case-control study [58] found positive associations.

For colorectal cancer risk, our meta-analysis found no positive results using random effects for SB, SSB or FJ intake. Despite having performed secondary analyses excluding outliers and having explained between-studies heterogeneity, results for the random-effect model remained non-significant. This is in a way consistent with results from a previous meta-analysis, which found no association between SSBs and colon cancer risk using a random-effects model [21]. On the other hand, a cohort study from 2014 found a positive association for an increase in $330 \mathrm{~mL} /$ day of SSBs [91]. Likewise, an Australian study that compared extreme categories of SSB intake $(\geq 200 \mathrm{~mL} /$ day versus $<6.7 \mathrm{~mL} /$ day $)$ showed positive results [54]. We included only one study assessing rectal cancer incidence [92]. Here, a separate analysis for women and men was performed. The majority of the results were not significant, and the only positive association was found for juice (fruit and vegetables) consumption in female participants.

In regard to esophageal cancers, publications included in this review were also part of a meta-analysis from 2014 [18]. This meta-analysis reported no association between SBs and esophageal adenocarcinoma and squamous cell carcinoma risk. After extracting separated data for SSB and ASB intake, we found similar results. Despite these observations, positive associations were found in a pooled analysis of US-based case-control studies. This study assessed the association between sugar dietary intake and Barret's esophagus incidence, a precursor for esophageal adenocarcinoma tumor [96]. Even though data from the included studies reported non-significant results for stomach cancer incidence, a Japanese cohort study observed that carbonated drinks and juices appeared to be related to an elevated risk of death from stomach cancer [97].

With respect to pancreatic cancer, we performed a meta-analysis for SBs, SSBs and ASBs. These associations, especially for SBs, tended to be positive but did not reach statistically significant levels using random effect models. These results go along with a recent meta-analysis from 2019 [19] which also showed no association between SB intake and pancreatic cancer risk. Besides that, a pooled analysis from 2012 [20] reported a 56\% higher risk of pancreatic cancer for males consuming $\geq 375 \mathrm{~mL} /$ day of SSBs compared to non-consumers. Likewise, a Swedish cohort [41] found a 93\% higher risk of pancreatic cancer incidence among those who consumed $\geq 500 \mathrm{~mL} /$ day of SSBs compared to nonconsumers. However, we performed a subgroup analysis taking into account beverage intake category (high vs. non- consumer), but no significant associations were observed (Table S1). In addition, only one study reported separate results for carbonated and noncarbonated SBs, but no significant results were shown [76].

For bladder cancer risk, 3 out of the 6 included case-control studies $[62,63,65]$ showed positive associations for highest versus lowest amounts of SB intake. However, the metaanalysis of these studies together with 2 other case-control studies [61,64] showed no significant associations. Despite performing a second analysis excluding one study that presented some serious bias, the results remained non-significant (Table S1). Hence, our meta-analysis of observational studies reported that SBs appeared to be unrelated to bladder cancer risk. It is not clear how SSBs, ASBs or FJs act in isolation as the evidence is limited.

With reference to prostate cancer, our meta-analysis demonstrated an $18 \%$ higher risk for SSBs comparing the highest with the lowest intake. Similarly, we found a small positive association for FJs (a 3\% higher risk). No associations were found for SBs, which may suggest that the role of ASBs might not be relevant. However, one study [23] reported a positive association between ASB intake and prostate cancer risk. 
Renal cell cancer appeared to be unrelated to SB consumption according to the metaanalysis results. We observed a high between-study heterogeneity $\left(\mathrm{I}^{2}=65.4 \%\right)$. However, not enough studies $(n=3)$ were included to perform subgroup analyses. Even so, Maclure and Willet [70] reported a significant positive association between highest versus lowest SB intake and renal cell cancer risk (RR: 2.6, 95\% CI: 1.4-4.8). More studies analyzing this association are required for further clarification.

The association between SSB consumption and both endometrial and ovarian cancer risk tended to be positive but did not reach statistically significant levels. One study stratified results by types of endometrial cancer (I and II) [39]. They reported positive associations between highest versus lowest SSB and SFJ consumption and endometrial type I cancer in post-menopausal women, but not in type II. These might be because subtypes may have different risk factors, even though evidence on this etiologic heterogeneity is quite limited [98]. Data from two studies [71,72] suggested that FJ intake might be a protective factor for cervical cancer. FJ consumption is often considered part of a healthy diet and lifestyle [99]. However, none of the mentioned studies [71,72] adjusted for such confounders. Thus, it is not clear if the protective effect was due to FJ intake or other factors. For epithelial ovarian cancer, one US study [75] stratified the results by caffeinated and noncaffeinated colas. Both results were positive statistically significant, but non-caffeinated colas showed a stronger association. Although this might suggest a protective effect of caffeine, a recent meta-analysis of prospective studies found no link between caffeine intake and ovarian cancer risk [100].

In respect of hepatobiliary cancers, data from the included studies showed a positive association with SB consumption, especially for gallbladder cancer, where the risk was doubled [49]. This might be explained by the detrimental association between sucrose/glycemic load and the increased risk of symptomatic gallstone disease [101], which is strongly correlated with gallbladder cancer [102]. Stepien et al. [28] showed slightly positive dose-response associations between SBs, ASBs or FJs and HCC incidence.

As regards hematologic cancers, no associations were found either for sugary or for artificially sweetened beverages, except for leukemia risk, for which one study [24] reported significant positive associations with ASBs. However, a recent review of clinical trials and observational studies observed no association between artificial sweeteners intake and both leukemia and non-Hodgkin lymphoma incidence [103].

The evidence is more limited regarding cancer of the oral cavity, pharynx, larynx, lung, thyroid, glioma and cutaneous myeloma. The available data mainly showed nonsignificant results for SB and FJ intake. Only one study from Montenegro indicated an inverse association between SB intake and laryngeal cancer risk [82]. However, the results from this study should be treated with caution as they presented some methodological inadequacies and its overall risk of bias was classified as 'serious risk' (Table S3). One case-control study from 1997 observed a strong positive association between small intestine cancer risk and SSBs (OR: 3.6, 95\% CI: 1.3-9.8), although further high quality evidence is needed [87]. One study that reported incidence of overall non-obesity-related cancers showed no association for SSBs but a positive association for ASBs [50]. Moreover, the largest of 3 studies [23,52,54] on overall obesity-related cancer risk showed positive associations with SFJs, SSBs and FJs, but not with ASB consumption [23]. Similarly, a meta-analysis of clinical trials and observational studies showed no association between artificial sweetener intake, body weight and different types of cancers [103]. Our findings are in accordance and we agree with the previous study [103] upon the uncertainty of the evidence that links artificial sweeteners with different types of cancer.

\subsection{Limitations of the Current Data}

To the best of our knowledge, this is the first systematic review to evaluate the isolated association between different groups of sweet beverages and cancer risk. Several limitations should be considered while interpreting our findings. Some studies included in this systematic review were difficult to compare due to their design (cohort and case- 
control studies), methodology, classification and categories of beverages intake. Therefore, it was a challenge to perform such comparisons. According to the ROBIN-E tool, cohort studies were at low-moderate risk of bias. As per the NOS, the case-control studies were at moderate risk of bias and 6 studies $[58,60,66,79,82,86]$ out of 37 presented serious methodology inadequacies. The number of publications included in most meta-analysis was relatively low (between 3 and 6). On this basis, the pooled effect size was calculated based on risk ratios of cohort and case-control studies together. Not having enough studies was a major limitation to perform subgroup analyses when high between-study heterogeneity was observed. Moreover, the small amount of studies may have been a potential source of unexplained heterogeneity [104]. We did not have enough data to perform subgroup analyses based on different population characteristics (e.g., sex, lifestyle factors or history of cancer). However, we did perform subgroup analyses based on geographical area.

The majority of the included participants were from the US or European countries. Hence, extrapolating our findings to other geographical areas may not be appropriate. We attempted to classify beverages into specific groups. However, some studies did not report precise information on this topic, which might have given rise to misclassifications. Similarly, we attempted to convert original exposure information into amounts of intake (mL/day) based on national data. Nevertheless, this was not possible in all studies which prohibited performance of a dose-response meta-analysis. Another limitation may be the measurement error in collecting dietary data since self-reported questionnaires were used. Moreover, in the longitudinal studies we were limited to the baseline estimation of beverages consumption, and there is a possibility that their consumption changed over time. It is suggested that the link between SSBs or FJs and cancer risk is possible due to their high glycemic indexes [13] and to obesity-inducing pathways [4]. However, these variables were not adequately integrated as confounders in all the studies. Indeed, glycemic index was only considered in one cohort [53]. Despite BMI being a common indicator of obesity and most studies considering it as confounder, only 4 of them $[35,40,52,54]$ adjusted for other obesity indicators such as waist circumference. Most of the studies assessed the association between consumption of SSB and common cancers such as breast, colorectal, prostate and pancreatic cancer. Data were more limited for FJs or ASBs and other types of cancers, especially non-obesity-related ones. FJ consumption may coexist with healthy habits, such as healthy diet or exercise [99]. Therefore, it would have been even better if some studies had adjusted their analysis for such variables. In fact, only 3 publications [52-54] used diet quality as a confounder.

\section{Conclusions}

The current meta-analysis of cohort and case-control studies indicated a statistically significant positive association between higher consumption of SSB and breast and prostate cancer incidence. As regard pre-menopausal breast cancer, results from cohort studies alone showed a significant association. Likewise, it showed a statistically significant positive link between high intakes of FJs and prostate cancer risk. Although the associations between other sweet beverages and other cancer types were also positive, they did not reach statistically significant levels. The small number of studies and cancer cases might have been a reason why we did not find statistically significant results for several cancer types. Study location (US/non-US, mostly European) did not appear to influence the results. Current evidence indicates that higher incidence of some cancers is related to a high consumption of SSBs. However, the evidence is limited to make recommendations regarding ASBs and FJs. This subject requires further investigation.

We encourage future research in this field to perform more separated analysis on SSB, ASB and FJ consumption. We believe it would be prudent to establish a homogeneous classification of beverages in order to better understand their role in carcinogenesis. We also recommend considering other obesity-related factors besides body mass index, such as waist circumference, glycemic index and quality of diet as confounders. We could not 
study the different roles of non-carbonated soft drinks (sport, fruit and tea-based drinks), sometimes used as healthier alternatives to carbonated drinks [105]. Therefore, it would be advisable for future studies to further explore this research area.

This systematic review supports the WCRF/AICR recommendations to limit sugary drinks consumption for cancer prevention [106] and to raise consumers' awareness of their low nutritional quality and high sugar content. We recommend replacement of sweet beverages with plain safe drinking water and infusions without added sugars as the main liquid source for body hydration. Even though some guidelines maintain that moderate consumption of FJs may be part of a healthy diet [107], FJs contain little or no dietary fiber and are positively associated with tooth decay in children [108]. Professional societies have recently recommended limiting children's FJ consumption as means of addressing the obesity epidemic [3]. Whole fruits and plain safe drinking water should also be affirmed as a healthier alternative to sweet beverages in adults. This would aim to promote the appropriate consumption of essential nutrients, to reduce intake of excessive sugars/calories and to therefore lower cardiometabolic disease and cancer incidences [109,110]. The increase in cancer [25], obesity [111] and type 2 diabetes [112] requires policy action. We recommend policymakers worldwide to consider (or continue with) taxation and marketing restriction for sweet beverages, especially SSBs.

Supplementary Materials: The following are available online at https:/ / www.mdpi.com/2072-6 643/13/2/516/s1, Table S1: Additional quantitative analysis for the association between sweet beverages and cancer risk; Table S2: Risk of bias in the included cohort studies according to Risk Of Bias In Non-randomized Studies_of Exposures (ROBINS-E); Table S3: Risk of bias in the included case-control studies, according to Newcastle-Ottawa Scale (NOS); Figure S1: Forest plot showing the pooled risk ratios with $95 \% \mathrm{CI}$ for cancer risk, comparing the highest vs. the lowest sugar-sweetened beverages (SSB) intake category; Figure S2: Forest plot showing pooled risk ratios with 95\% CI for cancer risk, comparing the highest vs. the lowest artificially and sugar-sweetened beverages (SB) intake category; Figure S3: Forest plot showing the pooled risk ratios with $95 \%$ CI for cancer risk, comparing the highest vs. the lowest fruit juice (FJ) intake category; Figure S4: Forest plot showing the pooled risk ratios with $95 \%$ CI for cancer risk, comparing the highest vs. the lowest artificially sweetened beverage (ASB) intake category.

Author Contributions: Conceptualization and design, R.Z.-R.; study selection, F.L., M.G.-L. and P.U.; data extraction, F.L., M.G.-L. and P.U.; data synthesis, F.L., M.G.-L. and P.U.; statistical analysis, I.d.V.; writing — original draft preparation, F.L., M.G.-L. and P.U.; writing—review and editing, J.C. and R.Z.-R. All authors have read and agreed to the published version of the manuscript.

Funding: This research was funded by the Institute of Health Carlos III through the grant CP15/00100 and PI18/00191 (cofounded by European Regional Development Fund. ERDF, a way to build Europe) and from the 201943-30-31 project, funded by La Marato de TV-3. We thank CERCA Program/Generalitat de Catalunya for the institutional support. M.G.-L. is grateful for the predoctoral scholarship PFIS (FI19/00185) from the Institute of Health Carlos III. J.C. is thankful for the CONACYT fellowship (ID 693636) from the Mexican Government. R.Z.-R. would like to thank the 'Miguel Servet' program (CP15/00100) from the Institute of Health Carlos III (cofounded by the European Social Fund (ESF)—ESF investing in your future).

Informed Consent Statement: Data was collected from available literature.

Data Availability Statement: Data for doing the meta-analysis is available in the manuscript's tables.

Conflicts of Interest: The authors declare no conflict of interest. The funders had no role in the design of the study; in the collection, analyses, interpretation of data; or in the writing and publishing of the manuscript. 


\section{References}

1. Welsh, J.A.; Lundeen, E.A.; Stein, A.D. The sugar-sweetened beverage wars: Public health and the role of the beverage industry. Curr. Opin. Endocrinol. Diabetes Obes. 2013, 20, 401-406. [CrossRef] [PubMed]

2. Sylvetsky, A.C.; Jin, Y.; Clark, E.J.; Welsh, J.A.; Rother, K.I.; Talegawkar, S.A. Consumption of Low-Calorie Sweeteners among Children and Adults in the United States. J. Acad. Nutr. Diet. 2017, 117, 441-448. [CrossRef] [PubMed]

3. Wojcicki, J.M.; Heyman, M.B. Reducing childhood obesity by eliminating $100 \%$ fruit juice. Am. J. Public Health 2012, 102, 1630-1633. [CrossRef] [PubMed]

4. Te Morenga, L.; Simonette, M.; Jim, M. Dietary sugars and body weight: Systematic review and meta-analyses of randomised controlled trials and cohort studies. BMJ 2013, 346, e7492. [CrossRef] [PubMed]

5. Imamura, F.; O'Connor, L.; Ye, Z.; Mursu, J.; Hayashino, Y.; Bhupathiraju, S.N.; Forouhi, N.G. Consumption of sugar sweetened beverages, artificially sweetened beverages, and fruit juice and incidence of type 2 diabetes: Systematic review, meta-analysis, and estimation of population attributable fraction. BMJ 2015, 351. [CrossRef]

6. Friedman, F.; Mozaffarian, D. Dietary and Policy Priorities for Cardiovascular Disease, Diabetes, and Obesity A Comprehensive Review. Circulation 2016, 133, 187-225. [CrossRef]

7. Calle, E.E.; Kaaks, R. Overweight, obesity and cancer: Epidemiological evidence and proposed mechanisms. Nat. Rev. Cancer 2004, 4, 579-591. [CrossRef]

8. Giovannucci, E.; Harlan, D.M.; Archer, M.C.; Bergenstal, R.M.; Gapstur, S.M.; Habel, L.A.; Pollak, M.; Regensteiner, J.G.; Yee, D. Diabetes and Cancer: A consensus report. Diabetes Care 2010, 33, 1674-1685. [CrossRef] [PubMed]

9. Elliott, S.S.; Keim, N.L.; Stern, J.S.; Teff, K.; Havel, P.J. Fructose, weight gain, and the insulin resistance syndrome. Am. J. Clin. Nutr. 2002, 76, 911-933. [CrossRef]

10. Lauby-Secretan, B.; Scoccianti, C.; Loomis, D.; Grosse, Y.; Bianchini, F.; Straif, K. Body Fatness and Cancer-Viewpoint of the IARC Working Group. N. Engl. J. Med. 2016, 375, 794-798. [CrossRef]

11. Giovannucci, E. Insulin, insulin-like growth factors and colon cancer: A review of the evidence. J. Nutr. 2001, 131, 3109S-3120S. [CrossRef]

12. Atkinson, F.S.; Foster-Powell, K.; Brand-Miller, J.C. International tables of glycemic index and glycemic load values: 2008. Diabetes Care 2008, 31, 2281-2283. [CrossRef] [PubMed]

13. Sieri, S.; Krogh, V. Dietary glycemic index, glycemic load and cancer: An overview of the literature. Nutr. Metab. Cardiovasc. Dis. 2017, 27, 18-31. [CrossRef] [PubMed]

14. Lerma-Cabrera, J.M.; Carvajal, F.; Lopez-Legarrea, P. Food addiction as a new piece of the obesity framework. Nutr. J. 2016, 15. [CrossRef]

15. Ruanpeng, D.; Thongprayoon, C.; Cheungpasitporn, W.; Harindhanavudhi, T. Sugar and artificially sweetened beverages linked to obesity: A systematic review and meta-analysis. QJM Int. J. Med. 2017, 110, 513-520. [CrossRef]

16. Soffritti, M.; Belpoggi, F.; Degli Esposti, D.; Lambertini, L. Aspartame induces lymphomas and leukaemias in rats. Eur. J. Oncol. 2005, 10, 107-116.

17. International Agency for Research on Cancer Monograph Working Group. Formaldehyde, 2-Butoxyethanol and 1-tertButoxypropan-2-ol. IARC Monogr. Eval. Carcinog. Risks Hum. 2006, 88, 1-478.

18. Boyle, P.; Koechlin, A.; Autier, P. Sweetened carbonated beverage consumption and cancer risk. Eur. J. Cancer Prev. 2014, 23, 481-490. [CrossRef]

19. Milajerdi, A.; Larijani, B.; Esmaillzadeh, A. Sweetened Beverages Consumption and Pancreatic Cancer: A Meta-Analysis. Nutr. Cancer 2019, 71, 375-384. [CrossRef]

20. Genkinger, J.M.; Li, R.; Spiegelman, D.; Anderson, K.E.; Albanes, D.; Bergkvist, L.; Bernstein, L.; Black, A.; Van Den Brandt, P.A.; English, D.R.; et al. Coffee, tea, and sugar-sweetened carbonated soft drink intake and pancreatic cancer risk: A pooled analysis of 14 cohort studies. Cancer Epidemiol. Biomark. Prev. 2012, 21, 305-318. [CrossRef]

21. Zhang, X.; Albanes, D.; Beeson, W.L.; Van Den Brandt, P.A.; Buring, J.E.; Flood, A.; Freudenheim, J.L.; Giovannucci, E.L.; Goldbohm, R.A.; Jaceldo-Siegl, K.; et al. Risk of colon cancer and coffee, tea, and sugar-sweetened soft drink intake: Pooled analysis of prospective cohort studies. J. Natl. Cancer Inst. 2010, 102, 771-783. [CrossRef] [PubMed]

22. Makarem, N.; Bandera, E.V.; Nicholson, J.M.; Parekh, N. Consumption of Sugars, Sugary Foods, and Sugary Beverages in Relation to Cancer Risk: A Systematic Review of Longitudinal Studies. Annu. Rev. Nutr. 2018, 38, 17-39. [CrossRef] [PubMed]

23. Chazelas, E.; Srour, B.; Desmetz, E.; Kesse-Guyot, E.; Julia, C.; Deschamps, V.; Druesne-Pecollo, N.; Galan, P.; Hercberg, S.; Latino-Martel, P.; et al. Sugary drink consumption and risk of cancer: Results from NutriNet-Santé prospective cohort. BMJ 2019, 366, 12408. [CrossRef]

24. Schernhammer, E.S.; Bertrand, K.A.; Birmann, B.M.; Sampson, L.; Willett, W.C.; Feskanich, D. Consumption of artificial sweetenerand sugar-containing soda and risk of lymphoma and leukemia in men and women. Am. J. Clin. Nutr. 2012, 96, 1419-1428. [CrossRef] [PubMed]

25. World Health Organization (WHO). Report on Cancer: Setting Priorities, Investing Wisely and Providing Care for All. Available online: https: / / apps.who.int/iris/handle/10665/330745 (accessed on 30 November 2020).

26. Bero, L.; Chartres, N.; Diong, J.; Fabbri, A.; Ghersi, D.; Lam, J.; Lau, A.; McDonald, S.; Mintzes, B.; Sutton, P.; et al. The risk of bias in observational studies of exposures (ROBINS-E) tool: Concerns arising from application to observational studies of exposures. Syst. Rev. 2018, 7, 242. [CrossRef] 
27. The Newcastle-Ottawa Scale (NOS) for Assessing the Quality of Nonrandomised Studies in Meta-Analyses. Available online: http:/ / www.ohri.ca/programs/clinical_epidemiology/oxford.asp (accessed on 2 November 2020).

28. Stepien, M.; Duarte-Salles, T.; Fedirko, V.; Trichopoulou, A.; Lagiou, P.; Bamia, C.; Overvad, K.; Tjønneland, A.; Hansen, L.; Boutron-Ruault, M.C.; et al. Consumption of soft drinks and juices and risk of liver and biliary tract cancers in a European cohort. Eur. J. Nutr. 2014, 55, 7-20. [CrossRef] [PubMed]

29. US Food and Drug Administration (FDA), Code of Federal Regulations Title 21. Available online: https://www.accessdata.fda. gov/scripts/cdrh/cfdocs/cfcfr/cfrsearch.cfm?fr=101.12 (accessed on 30 November 2020).

30. Australian Food Composition Database. Available online: https://www.foodstandards.gov.au/science/monitoringnutrients/ afcd/Pages/default.aspx (accessed on 30 November 2020).

31. Miles, F.L.; Neuhouser, M.L.; Zhang, Z.F. Concentrated sugars and incidence of prostate cancer in a prospective cohort. Proc. Int. Astron. Union 2018, 120, 703-710. [CrossRef] [PubMed]

32. USDA, Food Data Central. Available online: https://ndb.nal.usda.gov/download-datasets.html (accessed on 2 November 2020).

33. Viechtbauer, W. Conducting meta-analyses in R with the metafor. J. Stat. Softw. 2010, 36, 1-48. [CrossRef]

34. Ren, J.S.; Freedman, N.D.; Kamangar, F.; Dawsey, S.M.; Hollenbeck, A.R.; Schatzkin, A.; Abnet, C.C. Tea, coffee, carbonated soft drinks and upper gastrointestinal tract cancer risk in a large United States prospective cohort study. Eur. J. Cancer 2010, 46, 1873-1881. [CrossRef]

35. Drake, I.; Sonestedt, E.; Gullberg, B.; Ahlgren, G.; Bjartell, A.; Wallström, P.; Wirfält, E. Dietary intakes of carbohydrates in relation to prostate cancer risk: A prospective study in the Malmö Diet and Cancer cohort. Am. J. Clin. Nutr. 2012, 96, 1409-1418. [CrossRef]

36. Ellison, L.F. Tea and other beverage consumption and prostate cancer risk: A Canadian retrospective cohort study. Eur. J. Cancer Prev. 2000, 9, 125-130. [CrossRef] [PubMed]

37. Lee, J.E.; Giovannucci, E.; Smith-Warner, S.A.; Spiegelman, D.; Willett, W.C.; Curhan, G.C. Total fluid intake and use of individual beverages and risk of renal cell cancer in two large cohorts. Cancer Epidemiol. Biomarkers Prev. 2006, 15, 1204-1211. [CrossRef] [PubMed]

38. Ros, M.M.; Bas Bueno-de-Mesquita, H.B.; Büchner, F.L.; Aben, K.K.H.; Kampman, E.; Egevad, L.; Overvad, K.; Tjønneland, A.; Roswall, N.; Clavel-Chapelon, F.; et al. Fluid intake and the risk of urothelial cell carcinomas in the European Prospective Investigation into Cancer and Nutrition (EPIC). Int. J. Cancer 2011, 128, 2695-2708. [CrossRef] [PubMed]

39. Inoue-Choi, M.; Robien, K.; Mariani, A.; Cerhan, J.R.; Anderson, K.E. Sugar-sweetened beverage intake and the risk of type I and type II endometrial cancer among postmenopausal women. Cancer Epidemiol. Biomarkers Prev. 2013, 22, 2384-2394. [CrossRef] [PubMed]

40. McCullough, M.L.; Teras, L.R.; Shah, R.; Diver, W.R.; Gaudet, M.M.; Gapstur, S.M. Artificially and Sugar-Sweetened Carbonated Beverage Consumption Is Not Associated with Risk of Lymphoid Neoplasms in Older Men and Women. J. Nutr. 2014, 144, 2041-2049. [CrossRef] [PubMed]

41. Larsson, S.C.; Bergkvist, L.; Wolk, A. Consumption of sugar and sugar-sweetened foods and the risk of pancreatic cancer in a prospective study. Am. J. Clin. Nutr. 2006, 84, 1171-1176. [CrossRef]

42. Bao, Y.; Stolzenberg-Solomon, R.; Jiao, L.; Silverman, D.T.; Subar, A.F.; Park, Y.; Leitzmann, M.F.; Hollenbeck, A.; Schatzkin, A.; Michaud, D.S. Added sugar and sugar-sweetened foods and beverages and the risk of pancreatic cancer in the National Institutes of Health-AARP Diet and Health Study. Am. J. Clin. Nutr. 2008, 88, 431-440. [CrossRef] [PubMed]

43. Mueller, N.T.; Odegaard, A.; Anderson, K.; Yuan, J.M.; Gross, M.; Koh, W.P.; Pereira, M.A. Soft drink and juice consumption and risk of pancreatic cancer: The singapore chinese health study. Cancer Epidemiol. Biomarkers Prev. 2010, 19, 447-455. [CrossRef]

44. Nöthlings, U.; Murphy, S.P.; Wilkens, L.R.; Henderson, B.E.; Kolonel, L.N. Dietary glycemic load, added sugars, and carbohydrates as risk factors for pancreatic cancer: The Multiethnic Cohort Study. Am. J. Clin. Nutr. 2007, 86, 1495-1501. [CrossRef]

45. Navarrete-Muñoz, E.M.; Wark, P.A.; Romaguera, D.; Bhoo-Pathy, N.; Michaud, D.; Molina-Montes, E.; Tjønneland, A.; Olsen, A.; Overvad, K.; Boutron-Ruault, M.C.; et al. Sweet-beverage consumption and risk of pancreatic cancer in the European Prospective Investigation into Cancer and Nutrition (EPIC). Am. J. Clin. Nutr. 2016, 104, 760-768. [CrossRef]

46. Schernhammer, E.S.; Hu, F.B.; Giovannucci, E.; Michaud, D.S.; Colditz, G.A.; Stampfer, M.J.; Fuchs, C.S. Sugar-sweetened soft drink consumption and risk of pancreatic cancer in two prospective cohorts. Cancer Epidemiol. Biomarkers Prev. 2005, 14, 2098-2105. [CrossRef] [PubMed]

47. Dubrow, R.; Darefsky, A.S.; Freedman, N.D.; Hollenbeck, A.R.; Sinha, R. Coffee, tea, soda, and caffeine intake in relation to risk of adult glioma in the NIH-AARP Diet and Health Study. Cancer Causes Control 2012, 23, 757-768. [CrossRef] [PubMed]

48. Zamora-Ros, R.; Béraud, V.; Franceschi, S.; Cayssials, V.; Tsilidis, K.K.; Boutron-Ruault, M.C.; Weiderpass, E.; Overvad, K.; Tjønneland, A.; Eriksen, A.K.; et al. Consumption of fruits, vegetables and fruit juices and differentiated thyroid carcinoma risk in the European Prospective Investigation into Cancer and Nutrition (EPIC) study. Int. J. Cancer 2018, 142, 449-459. [CrossRef] [PubMed]

49. Larsson, S.C.; Giovannucci, E.L.; Wolk, A. Sweetened Beverage Consumption and Risk of Biliary Tract and Gallbladder Cancer in a Prospective Study. J. Natl. Cancer Inst. 2016, 108. [CrossRef]

50. Bassett, J.K.; Milne, R.L.; English, D.R.; Giles, G.G.; Hodge, A.M. Consumption of sugar-sweetened and artificially sweetened soft drinks and risk of cancers not related to obesity. Int. J. Cancer 2020, 146, 3329-3334. [CrossRef] 
51. Hirvonen, T.; Mennen, L.I.; de Bree, A.; Castetbon, K.; Galan, P.; Bertrais, S.; Arnault, N.; Hercberg, S. Consumption of Antioxidant-Rich Beverages and Risk for Breast Cancer in French Women. Ann. Epidemiol. 2006, 16, 503-508. [CrossRef]

52. Makarem, N.; Bandera, E.V.; Lin, Y.; Jacques, P.F.; Hayes, R.B.; Parekh, N. Consumption of sugars, sugary foods, and sugary beverages in relation to adiposity-related cancer risk in the framingham offspring cohort (1991-2013). Cancer Prev. Res. 2018, 11, 347-358. [CrossRef]

53. Romanos-Nanclares, A.; Toledo, E.; Gardeazabal, I.; Jiménez-Moleón, J.J.; Martínez-González, M.A.; Gea, A. Sugar-sweetened beverage consumption and incidence of breast cancer: The Seguimiento Universidad de Navarra (SUN) Project. Eur. J. Nutr. 2019, 58, 2875-2886. [CrossRef]

54. Hodge, A.M.; Bassett, J.K.; Milne, R.L.; English, D.R.; Giles, G.G. Consumption of sugar-sweetened and artificially sweetened soft drinks and risk of obesity-related cancers. Public Health Nutr. 2018, 21, 1618-1626. [CrossRef]

55. Nomura, S.J.O.; Dash, C.; Rosenberg, L.; Yu, J.; Palmer, J.R.; Adams-Campbell, L.L. Adherence to diet, physical activity and body weight recommendations and breast cancer incidence in the Black Women's Health Study. Int. J. Cancer 2016, 139, $2738-2752$. [CrossRef]

56. Pacheco, L.S.; Anderson, C.A.M.; Lacey, J.V.; Giovannucci, E.L.; Lemus, H.; Araneta, M.R.G.; Sears, D.D.; Talavera, G.A.; Martinez, M.E. Sugar-sweetened beverages and colorectal cancer risk in the California Teachers Study. PLoS ONE 2019, 14, e0223638. [CrossRef] [PubMed]

57. Chandran, U.; McCann, S.E.; Zirpoli, G.; Gong, Z.; Lin, Y.; Hong, C.C.; Ciupak, G.; Pawlish, K.; Ambrosone, C.B.; Bandera, E.V. Intake of energy-dense foods, fast foods, sugary drinks, and breast cancer risk in African American and European American women. Nutr. Cancer 2014, 66, 1187-1199. [CrossRef] [PubMed]

58. Marzbani, B.; Nazari, J.; Najafi, F.; Marzbani, B.; Shahabadi, S.; Amini, M.; Moradinazar, M.; Pasdar, Y.; Shakiba, E.; Amini, S. Dietary patterns, nutrition, and risk of breast cancer: A case-control study in the west of Iran. Epidemiol. Health 2019, 41, e2019003. [CrossRef] [PubMed]

59. Mayne, S.T.; Risch, H.A.; Dubrow, R.; Chow, W.H.; Gammon, M.D.; Vaughan, T.L.; Borchardt, L.; Schoenberg, J.B.; Stanford, J.L.; West, A.B.; et al. Carbonated soft drink consumption and risk of esophageal adenocarcinoma. J. Natl. Cancer Inst. 2006, $98,72-75$. [CrossRef]

60. Bruemmer, B.; White, E.; Vaughan, T.L.; Cheney, C.L. Fluid intake and the incidence of bladder cancer among middle-aged men and women in a three-county area of Western Washington. Nutr. Cancer 1997, 29, 163-168. [CrossRef]

61. De Stefani, E.; Boffetta, P.; Deneo-Pellegrini, H.; Correa, P.; Ronco, A.L.; Brennan, P.; Ferro, G.; Acosta, G.; Mendilaharsu, M. Non-alcoholic beverages and risk of bladder cancer in Uruguay. BMC Cancer 2007, 7. [CrossRef] [PubMed]

62. Hemelt, M.; Hu, Z.; Zhong, Z.; Xie, L.P.; Wong, Y.C.; Tam, P.C.; Cheng, K.K.; Ye, Z.; Bi, X.; Lu, Q.; et al. Fluid intake and the risk of bladder cancer: Results from the South and East China case-control study on bladder cancer. Int. J. Cancer 2010, 127, 638-645. [CrossRef] [PubMed]

63. Radosavljević, V.; Janković, S.; Marinković, J.; Djokić, M. Fluid intake and bladder cancer. A case control study. Neoplasma 2003, 50, 234-238. [PubMed]

64. Turati, F.; Bosetti, C.; Polesel, J.; Zucchetto, A.; Serraino, D.; Montella, M.; Libra, M.; Galfano, A.; La Vecchia, C.; Tavani, A. Coffee, Tea, Cola, and Bladder Cancer Risk: Dose and Time Relationships. Urology 2015, 86, 1179-1184. [CrossRef]

65. Wang, J.; Wu, X.; Kamat, A.; Grossman, H.B.; Dinney, C.P.; Lin, J. Fluid intake, genetic variants of UDP-glucuronosyltransferases, and bladder cancer risk. Br. J. Cancer 2013, 108, 2372-2380. [CrossRef]

66. Jain, M.G.; Hislop, G.T.; Howe, G.R.; Burch, J.D.; Ghadirian, P. Alcohol and other beverage use and prostate cancer risk among Canadian men. Int. J. Cancer 1998, 78, 707-711. [CrossRef]

67. Sharpe, C.R.; Siemiatycki, J. Consumption of non-alcoholic beverages and prostate cancer risk. Eur. J. Cancer Prev. 2002, 11, 497-501. [CrossRef] [PubMed]

68. Hu, J.; Mao, Y.; DesMeules, M.; Csizmadi, I.; Friedenreich, C.; Mery, L. Total fluid and specific beverage intake and risk of renal cell carcinoma in Canada. Cancer Epidemiol. 2009, 33, 355-362. [CrossRef]

69. McLaughlin, C.C.; Mahoney, M.C.; Nasca, P.C.; Metzger, B.B.; Baptiste, M.S.; Field, N.A. Breast cancer and methylxanthine consumption. Cancer Causes Control 1992, 3, 175-178. [CrossRef]

70. Maclure, M.; Willett, W. A case-control study of diet and risk of renal adenocarcinoma. Epidemiology 1990, 1, 430-440. [CrossRef]

71. Herrero, R.; Potischman, N.; Brinton, L.A.; Reeves, W.C.; Brenes, M.M.; Tenorio, F.; De Britton, R.C.; Gaitan, E. A case-control study of nutrient status and invasive cervical cancer: I. Dietary indicators. Am. J. Epidemiol. 1991, 134, 1335-1346. [CrossRef] [PubMed]

72. Verreault, R.; Chu, J.; Mandelson, M.; Shy, K. A case-control study of diet and invasive cervical cancer. Int. J. Cancer 1989, 43, 1050-1054. [CrossRef]

73. King, M.G.; Olson, S.H.; Paddock, L.; Chandran, U.; Demissie, K.; Lu, S.E.; Parekh, N.; Rodriguez-Rodriguez, L.; Bandera, E.V. Sugary food and beverage consumption and epithelial ovarian cancer risk: A population-based case-control study. BMC Cancer 2013, 13, 94. [CrossRef] [PubMed]

74. Leung, A.C.Y.; Cook, L.S.; Swenerton, K.; Gilks, B.; Gallagher, R.P.; Magliocco, A.; Steed, H.; Köbel, M.; Nation, J.; Brooks-Wilson, A.; et al. Tea, coffee, and caffeinated beverage consumption and risk of epithelial ovarian cancers. Cancer Epidemiol. 2016, 45, 119-125. [CrossRef] 
75. Yoon, J.S.; Kristal, A.R.; Wicklund, K.G.; Cushing-Haugen, K.L.; Rossing, M.A. Coffee, tea, colas, and risk of epithelial ovarian cancer. Cancer Epidemiol. Biomarkers Prev. 2008, 17, 712-716. [CrossRef]

76. Chan, J.M.; Wang, F.; Holly, E.A. Sweets, sweetened beverages, and risk of pancreatic cancer in a large population-based case-control study. Cancer Causes Control 2009, 20, 835-846. [CrossRef] [PubMed]

77. Gallus, S.; Turati, F.; Tavani, A.; Polesel, J.; Talamini, R.; Franceschi, S.; La Vecchia, C. Soft drinks, sweetened beverages and risk of pancreatic cancer. Cancer Causes Control 2011, 22, 33-39. [CrossRef] [PubMed]

78. Gold, E.B.; Gordis, L.; Diener, M.D.; Seltser, R.; Boitnott, J.K.; Bynum, T.E.; Hutcheon, D.F. Diet and other risk factors for cancer of the pancreas. Cancer 1985, 55, 460-467. [CrossRef]

79. Lyon, J.L.; Mahoney, A.W.; French, T.K.; Moser, R. Coffee consumption and the risk of cancer of the exocrine pancreas: A case-control study in a low-risk population. Epidemiology 1992, 3, 164-170. [CrossRef]

80. Potischman, N.; Coates, R.J.; Swanson, C.A.; Carroll, R.J.; Daling, J.R.; Brogan, D.R.; Gammon, M.D.; Midthune, D.; Curtin, J.; Brinton, L.A. Increased risk of early-stage breast cancer related to consumption of sweet foods among women less than age 45 in the United States. Cancer Causes Control 2002, 13, 937-946. [CrossRef] [PubMed]

81. Mack, T.; Yu, M.; Henderson, B. Pancreas cancer and smoking, beverage consumption, and past medical history. J. Natl. Cancer Inst. 1986, 76, 49-60.

82. Zvrko, E.; Gledović, Z.; Ljaljević, A. Risk factors for laryngeal cancer in Montenegro. Arh. Hig. Rada Toksikol. 2008, 59, 11-18. [CrossRef]

83. Lissowska, J.; Pilarska, A.; Pilarski, P.; Samolczyk-Wanyura, D.; Piekarczyk, J.; Bardin-Mikolajczak, A.; Zatonski, W.; Herrero, R.; Muňoz, N.; Franceschi, S. Smoking, alcohol, diet, dentition and sexual practices in the epidemiology of oral cancer in Poland. Eur. J. Cancer Prev. 2003, 12, 25-33. [CrossRef] [PubMed]

84. Kreimer, A.R.; Randi, G.; Herrero, R.; Castellsagué, X.; La Vecchia, C.; Franceschi, S. Diet and body mass, and oral and oropharyngeal squamous cell carcinomas: Analysis from the IARC multinational case-control study. Int. J. Cancer 2006, 118, 2293-2297. [CrossRef] [PubMed]

85. Vinceti, M.; Bonvicini, F.; Pellacani, G.; Sieri, S.; Malagoli, C.; Giusti, F.; Krogh, V.; Bergomi, M.; Seidenari, S. Food intake and risk of cutaneous melanoma in an Italian population. Eur. J. Clin. Nutr. 2008, 62, 1351-1354. [CrossRef]

86. Luqman, M.; Javed, M.M.; Daud, S.; Raheem, N.; Ahmad, J.; Khan, A.U.H. Risk factors for lung cancer in the Pakistani population. Asian Pacific J. Cancer Prev. 2014, 15, 3035-3039. [CrossRef]

87. Wu, A.H.; Yu, M.C.; Mack, T.M. Smoking, alcohol use, dietary factors and risk of small intestinal adenocarcinoma. Int. J. Cancer 1997, 70, 512-517. [CrossRef]

88. Bener, A.; Moore, M.A.; Ali, R.; El Ayoubi, H.R. Impacts of family history and lifestyle habits on colorectal cancer risk: A case-control study in Qatar. Asian Pacific J. Cancer Prev. 2010, 11, 963-968.

89. Mahfouz, E.M.; Sadek, R.R.; Abdel-Latief, W.M.; Mosallem, F.A.H.; Hassan, E.E. The role of dietary and lifestyle factors in the development of colorectal cancer: Case control study in Minia, Egypt. Cent. Eur. J. Public Health 2014, 22, 215-222. [CrossRef] [PubMed]

90. Tayyem, R.F.; Bawadi, H.A.; Shehadah, I.; Bani-Hani, K.E.; Takruri, H.; Al-Jaberi, T.; Heath, D.D. Fast foods, sweets and beverage consumption and risk of colorectal cancer: A case-control study in Jordan. Asian Pacific J. Cancer Prev. 2018, 19, 261-269. [CrossRef]

91. Theodoratou, E.; Farrington, S.M.; Tenesa, A.; McNeill, G.; Cetnarskyj, R.; Korakakis, E.; Din, F.V.N.; Porteous, M.E.; Dunlop, M.G.; Campbell, H. Associations between dietary and lifestyle risk factors and colorectal cancer in the Scottish population. Eur. J. Cancer Prev. 2014, 23, 8-17. [CrossRef]

92. Murtaugh, M.A.; Ma, K.N.; Caan, B.J.; Slattery, M.L. Association of fluids from beverages with risk of rectal cancer. Nutr. Cancer 2004, 49, 25-31. [CrossRef]

93. Ibiebele, T.I.; Hughes, M.C.; O’Rourke, P.; Webb, P.M.; Whiteman, D.C. Cancers of the esophagus and carbonated beverage consumption: A population-based case-control study. Cancer Causes Control 2008, 19, 577-584. [CrossRef]

94. Turati, F.; Dalmartello, M.; Bravi, F.; Serraino, D.; Augustin, L.; Giacosa, A.; Negri, E.; Levi, F.; Vecchia, C. La Adherence to the world cancer research fund/american institute for cancer research recommendations and the risk of breast cancer. Nutrients $\mathbf{2 0 2 0}$ 12, 607. [CrossRef] [PubMed]

95. Chandran, U.; Hirshfield, K.M.; Bandera, E.V. The role of anthropometric and nutritional factors on breast cancer risk in African-American women. Public Health Nutr. 2012, 15, 738-748. [CrossRef] [PubMed]

96. Li, N.; Petrick, J.L.; Steck, S.E.; Bradshaw, P.T.; McClain, K.M.; Niehoff, N.M.; Engel, L.S.; Shaheen, N.J.; Corley, D.A.; Vaughan, T.L.; et al. Dietary sugar/starches intake and Barrett's esophagus: A pooled analysis. Eur. J. Epidemiol. 2017, 32, 1007-1017. [CrossRef] [PubMed]

97. Khan, M.M.H.; Goto, R.; Kobayashi, K.; Suzumura, S.; Nagata, Y.; Sonoda, T.; Sakauchi, F.; Washio, M.; Mori, M. Dietary habits and cancer mortality among middle aged and older Japanese living in Hokkaido, Japan by cancer site and sex. Asian Pacific J. Cancer Prev. 2004, 5, 58-65.

98. Yang, H.P.; Wentzensen, N.; Trabert, B.; Gierach, G.L.; Felix, A.S.; Gunter, M.J.; Hollenbeck, A.; Park, Y.; Sherman, M.E.; Brinton, L.A. Endometrial cancer risk factors by 2 main histologic subtypes. Am. J. Epidemiol. 2013, 177, 142-151. [CrossRef]

99. O'Neil, C.E.; Nicklas, T.A.; Rampersaud, G.C.; Fulgoni, V.L. 100\% Orange juice consumption is associated with better diet quality, improved nutrient adequacy, decreased risk for obesity, and improved biomarkers of health in adults: National Health and Nutrition Examination Survey, 2003-2006. Nutr. J. 2012, 11. [CrossRef] 
100. Salari-Moghaddam, A.; Milajerdi, A.; Surkan, P.J.; Larijani, B.; Esmaillzadeh, A. Caffeine, Type of Coffee, and Risk of Ovarian Cancer: A Dose-Response Meta-Analysis of Prospective Studies. J. Clin. Endocrinol. Metab. 2019, 104, 5349-5359. [CrossRef]

101. Tsai, C.J.; Leitzmann, M.F.; Willett, W.C.; Giovannucci, E.L. Glycemic load, glycemic index, and carbohydrate intake in relation to risk of cholecystectomy in women. Gastroenterology 2005, 129, 105-112. [CrossRef]

102. Randi, G.; Franceschi, S.; La Vecchia, C. Gallbladder cancer worldwide: Geographical distribution and risk factors. Int. J. Cancer 2006, 118, 1591-1602. [CrossRef] [PubMed]

103. Toews, I.; Lohner, S.; Küllenberg De Gaudry, D.; Sommer, H.; Meerpohl, J.J. Association between intake of non-sugar sweeteners and health outcomes: Systematic review and meta-analyses of randomised and non-randomised controlled trials and observational studies. BMJ 2019, 364. [CrossRef] [PubMed]

104. Ruppar, T. Meta-analysis: How to quantify and explain heterogeneity? Eur. J. Cardiovasc. Nurs. 2020, 19, 646-652. [CrossRef]

105. Bucher, T.; Siegrist, M. Children's and parents' health perception of different soft drinks. Br. J. Nutr. 2015, 113, 526-535. [CrossRef]

106. World Cancer Research Fund/American Institute for Cancer Research. Diet, Nutrition, Physical Activity and Cancer: A Global Perspective A Summary of the Third Expert Report. Available online: https:/ /www.wcrf.org/dietandcancer/recommendations/ limit-sugar-sweetened-drinks (accessed on 15 December 2020).

107. Heyman, M.B.; Abrams, S.A. Fruit Juice in Infants, Children, and Adolescents: Current Recommendations. Am. Acad. Pediatrics 2017, 139, 20170967. [CrossRef] [PubMed]

108. Salas, M.M.S.; Nascimento, G.G.; Vargas-Ferreira, F.; Tarquinio, S.B.C.; Huysmans, M.C.D.N.J.M.; Demarco, F.F. Diet influenced tooth erosion prevalence in children and adolescents: Results of a meta-analysis and meta-regression. J. Dent. 2015, 43, 865-875. [CrossRef] [PubMed]

109. Pan, A.; Malik, V.S.; Schulze, M.B.; Manson, J.A.E.; Willett, W.C.; Hu, F.B. Plain-water intake and risk of type 2 diabetes in young and middle-aged women. Am. J. Clin. Nutr. 2012, 95, 1454-1460. [CrossRef] [PubMed]

110. Aune, D.; Giovannucci, E.; Boffetta, P.; Fadnes, L.T.; Keum, N.N.; Norat, T.; Greenwood, D.C.; Riboli, E.; Vatten, L.J.; Tonstad, S. Fruit and vegetable intake and the risk of cardiovascular disease, total cancer and all-cause mortality-A systematic review and dose-response meta-analysis of prospective studies. Int. J. Epidemiol. 2017, 46, 1029-1056. [CrossRef] [PubMed]

111. World Health Organization. Obesity and Overweight. Available online: https://www.who.int/news-room/fact-sheets/detail/ obesity-and-overweight (accessed on 23 January 2020).

112. Wild, S.; Bchir, M.B.; Roglic, G.; Green, A.; Sci, M.; Sicree, R.; King, H. Global Prevalence of Diabetes Estimates for the year 2000 and projections for 2030. Diabetes Care 2004, 27, 1047-1053. [CrossRef] [PubMed] 